\title{
Factorization of differential expansion for antiparallel double-braid knots
}

\section{A. Morozov}

ITEP,

Bol. Cheremushkinskaya, 25, Moscow 117218, Russia

Institute for Information Transmission Problems, Bol. Karetny, 19 (1), Moscow 127994, Russia

National Research Nuclear University MEPhI,

Kashirskoe sh., 31, Moscow 115409, Russia

E-mail: morozov@itep.ru

ABSTRACT: Continuing the quest for exclusive Racah matrices, which are needed for evaluation of colored arborescent-knot polynomials in Chern-Simons theory, we suggest to extract them from a new kind of a double-evolution - that of the antiparallel double-braids, which is a simple two-parametric family of two-bridge knots, generalizing the one-parametric family of twist knots. In the case of rectangular representations $R=\left[r^{s}\right]$ we found an evidence that the corresponding differential expansion miraculously factorizes and can be obtained from that for the twist knots. This reduces the problem of rectangular exclusive Racah to constructing the answers for just a few twist knots. We develop a recent conjecture on the structure of differential expansion for the simplest members of this family (the trefoil and the figure-eight knot) and provide the exhaustive answer for the first unknown case of $R=$ [33]. The answer includes HOMFLY of arbitrary twist and double-braid knots and Racah matrices $\bar{S}$ and $S$ - what allows to calculate [33]-colored polynomials for arbitrary arborescent (double-fat) knots. For generic rectangular representations fully described are only the contributions of the single-floor pyramids. One step still remains to be done.

KeYwords: Topological Field Theories, Chern-Simons Theories

ArXiv ePrint: 1606.06015 


\section{Contents}

1 Introduction 1

2 Exclusive Racah matrices from double evolutions 4

3 Fundamental representation 5

4 Twist knots for $R=[22]$

5 Factorization of differential expansion for double-braids $\quad 8$

6 Other rectangular representations $R=\left[r^{s}\right] \quad 10$

7 On the structure of $\mathcal{F}_{\mathcal{A}, \mathcal{B}}^{(m)} \quad 11$

$\begin{array}{lll}7.1 & \text { Already known cases } & 11\end{array}$

7.2 The first unknown case: $R=[33] \quad 12$

$\begin{array}{lll}7.3 & \text { On nullification for the unknot } & 14\end{array}$

$\begin{array}{lll}7.4 & \text { Eigenvalues } & 15\end{array}$

$\begin{array}{ll}7.5 & \text { Single-floor pyramids } \\ & 16\end{array}$

$\begin{array}{lll}7.6 & \text { Other floors } & 17\end{array}$

8 Conclusion $\quad 18$

A Exclusive Racah matrix $\bar{S}^{[3,3]} \quad 19$

\section{Introduction}

Construction of knot polynomials [1-8] is currently at the front-line of modern theoretical physics, because this is an exactly solvable problem in quantum field theory, which remains unsolved for years, despite tremendous effort by many distinguished researchers. Far ago it was reformulated in terms of Chern-Simons theory [9]-[17], where (at least for the knots in $S^{3}$ ) it is basically reduced to a free-field calculation, and a formal answer is provided [18][32] in terms of representation theory of quantum groups. This, however, does not help to obtain explicit answers, except for the simplest situations, covered by the well-known databases [33-35]. The problem is that group-theory methods themselves are undeveloped, moreover, the crucial quantities, starting from Racah matrices, are ambiguously defined in most interesting cases and thus do not attract attention of pure mathematicians. The ambiguities drop out of the final answers for knot polynomials, but intermediate steps involve less-invariant objects - what can in fact be interpreted as a kind of a new gauge invariance of some effective field theory [36, 37], arising on the way from the fundamental 
Chern-Simons to Wilson-loop observables. This somewhat unexpected twist of the story makes it even more similar to "real" Yang-Mills theories, like QCD, and confirms expectations that the study of exactly-solvable models can shed light on the more complicated physical problems.

Recent revival of interest and new fast progress in $2 d$ conformal theories (CFT) [38][57] attracted new attention to $3 d$ Chern-Simons, which is its closest relative - and naturally caused a progress there, which is, however, not so spectacular yet. The difference is that knot polynomials are exact non-perturbative quantities, associated with modular transformations of non-perturbative conformal blocks, which are still far from being wellstudied in conformal theory as well. Fortunately, modular transformations are simpler than conformal blocks themselves, and one can proceed with knot polynomials even when conformal-block issues remain unsolved. Recent achievements in this direction are largely based on the new version [58]-[77] of the Reshetikhin-Turaev (RT) formalism, when quantum $\mathcal{R}$-matrices in the space of representations, rather than in representation spaces, and Clebsh-Gordan coefficients are substituted by Racah and matrices (and their more complicated convolutions, known as mixing matrices). An early example of the strength of such approach was provided by the celebrated Rosso-Jones formula [78]-[93], which fully solves the problem of colored knot polynomials for a distinguished case of torus knots. However, despite a considerable progress, made in above references, nothing comparably impressive is yet achieved beyond torus knots - the problem turns to be extremely complicated. A new hope appeared with the introduction of the special class of double-fat knots in [94], where knot polynomials are presumably made from monodromy matrices of 4-point conformal blocks - and thus the CFT methods can be directly applied. This class is rather rich, in includes all the two-bridge and pretzel knots, moreover, it appeared to coincide with the arborescent knots, well known in mathematical literature [95, 96]. In general such knots are made by contractions of "fingers" and "propagators", with just four lines/strands inside, and their knot polynomials can be considered as correlators in some new effective field theory [36, 37], which is in fact a gauge theory, as we already mentioned. What is needed for arborescent knots is just a pair of Racah matrices,

$$
\begin{array}{llll}
S: & ((\bar{R} \otimes R) \otimes R \longrightarrow R) & \longrightarrow & (\bar{R} \otimes(R \otimes R) \longrightarrow R) \\
\bar{S}: & ((R \otimes \bar{R}) \otimes R \longrightarrow R) \longrightarrow(R \otimes(\bar{R} \otimes R) \longrightarrow R)
\end{array}
$$

called exclusive, to distinguish them from exclusive ones, where the "final state" is arbitrary representation $Q \in R \otimes R \otimes \bar{R}$, not just $R$. The problem, however, is that they are needed in arbitrary representation $R$, if one wants to calculate $R$-colored knot polynomials. Modern group theory is incapable to provide the answers beyond pure symmetric and antisymmetric representations [97-100] - the record results, obtained by various direct methods are for $R=[2,1]$ [101] and $R=[2,2]$ [102] (see also [103] and [104] for some inclusive Racah matrices for $R=[3,1]$ and $R=[2,2]$ respectively). Further progress on these lines seems to be beyond the current computer capacities.

However, the answers, when known, are pretty simple, and it is clear that they can be found/guessed by some other, indirect methods. One of the obvious ideas is to extract the 
Racah matrices from the answers for some relatively simple knots and then apply them to calculations of generic (at least, arborescent) knots. An illustration of this idea was already given in [102] with the example of representation $R=$ [22], where $S$ and $\bar{S}$ were extracted from exclusive 3 -strand Racah matrices, previously found from the difficult direct calculation in [104], using the two-parametric family, which is simultaneously arborescent and 3-strand. Despite impressive, this example just expands the small piece of knowledge about a given $R$ into a much bigger knowledge — but only about the same $R$. Given problems with higher $R$, this is not enough.

An example of a very different kind was provided in [105]. One of the recent discoveries about knot polynomials is their spectacular internal structure, known as differential expansion (DE) [106]-[110]. It goes back to discovery of "differentials" in [111], based on the understanding of Khovanov-Rozansky calculus [112-131] — a conceptually important alternative to RT approach. DE somehow lies at the intersection of two different formalisms and, non-surprisingly, is a very powerful idea - unfortunately, underestimated and undeveloped. Still, whenever applied, it proves effective. In fact, the matrices $S$ and $\bar{S}$ for all (anti)symmetric representations in [97-100] were obtained as generalization of the answers for the figure-eight $4_{1}$ and other twist knots in [106] and [107] — which were originally guessed from the study of what later became the differential expansion. The suggestion of [105] was to repeat this trick on the new level of knowledge: to look at newly available results, reformulate them in terms of differential expansion and then, hopefully, generalize - producing absolutely new knowledge, or at least conjectures, about the unknown. In this respect [105] was a success: the Rosso-Jones answers for the trefoil for all rectangular representations $R$ were reformulated in terms of differential expansion, what allowed to conjecture the rectangularly-colored HOMFLY for figure eight knot and, most important, to conjecture the general shape of differential expansion in this case for all defect-zero [110] knots. The purpose of this paper is to further built on this success and make a new step towards finding the matrices $\bar{S}$ and $S$ for arbitrary rectangular representations. In fact just one more guess remains to be done on this way after the present paper - but first an independent examination of the already made conjectures is highly desirable.

Since [108] it is known, that differential expansion is much simpler for antiparallel than antiparallel braids, i.e. for twist rather than torus knots (even 2-strand). The latter have non-vanishing defect [110] and instead possess non-trivial next levels of DE [108], i.e. an additional structure, which still needs to be understood. Here we concentrate on the simpler - antiparallel - story, where defect is always zero and the structure of DE is fully described in [105]. We demonstrate that it exhibits a new factorization property, relating expansion coefficients for multi-braid knots through those for twist knots. This opens a way to explicitly describe a double evolution, extract exclusive Racah matrices $S$ and $\bar{S}$ by a variation of the idea in [104] and apply the technique of [94]-[104] to find HOMFLY polynomials for arbitrary arborescent knots. in arbitrary rectangular representation $R=$ $r^{s}$. The success of this program - once factorization is discovered — depends on the possibility to guess the general shape of the DE coefficients for twist knots, which hopefully will be possible in the near future. 
Generalization to non-rectangular diagrams faces additional (perhaps, related) problems: the structure of DE is more sophisticated, even for trefoil and the figure eight, and non-trivial multiplicities arise, turning Racah matrices in more sophisticated operators, which do not possess any canonical matrix form. Moreover, additional care is needed [36, 37] in this case to formulate the arborescent calculus of [94] for multi-finger knots - the interaction vertices in the corresponding effective "field theory" are also not canonically defined (or "non-local"). Thus non-rectangular case will be further elaborated on elsewhere.

Despite there is hardly any need to advertise the long-standing problem of calculating at least some non-symmetrically-colored HOMFLY for at least some knots - any result here is a breakthrough - we begin in section 2 from explaining that the particular twoparametric family of antiparallel double-braids is especially important at the present stage of knowledge: if found, it provides exclusive Racah matrices and thus allows to calculate for arbitrary arborescent knots, what is already quite something. The idea is further illustrated by the example of the fundamental representation in section 3 . We switch to DE only in section 4, where the coefficients of the expansion, structured in [105], are found for the twist knots, by applying the evolution method [107] to the known [22]-coloredHOMFLY from [104]. The culmination comes in section 5, where with available examples we discover and illustrate the factorization property of DE for antiparallel double braids and reproduce from the approach of section 2 the exclusive Racah matrices $\bar{S}_{22}$, recently found from a very different double evolution in [102]. In section 6 we make a formal conjecture, that this property holds for arbitrary rectangular representations. Given all the recent achievements after [94] this basically reduces rectangular arborescent calculus to finding the DE coefficients in [105] for the rather simple one-parametric family of twist knots. For them a separate conjecture is needed, of which we cook up just a prototype in section 7: we make it in full generality for the single-floor pyramids. At two floors we consider only the first unknown example of $R=$ [33]. Further generalizations look rather straightforward, but are left for the future work.

Throughout the text we use the standard notation $\{x\}=x-x^{-1}$ and $[n]=\frac{\left\{q^{n}\right\}}{\{q\}}$.

\section{Exclusive Racah matrices from double evolutions}

In [102] exclusive Racah matrices $S$ were extracted from the double evolution family of 3-strand knots: they diagonalize the double evolution matrix. Then $\bar{S}$ is obtained from

$$
\bar{S}=\bar{T}^{-1} S T^{-1} S^{\dagger} \bar{T}^{-1}
$$

with diagonal matrices $T$ and $\bar{T}$, made from the eigenvalues of the relevant $\mathcal{R}$-matrices. Instead, the double evolution family of double-braid two-bridge knots defines $\bar{S}$ directly: in the notation of [94]

$$
H_{R}^{\{m, n\}}=d_{R} \cdot\left(\bar{S} \bar{T}^{2 m} \bar{S} \bar{T}^{2 n} \bar{S}\right)_{\emptyset \emptyset}=\sum_{\bar{X}, \bar{Y} \in R \otimes \bar{R}} d_{R} \sqrt{d_{\bar{X}} d_{\bar{Y}}} \lambda_{\bar{X}}^{2 m} \lambda_{\bar{Y}}^{2 n} \bar{S}_{\bar{X} \bar{Y}}
$$

and $S$ is then extracted from (2.1) as a diagonalizing matrix of $\bar{T} \bar{S} \bar{T}$. 


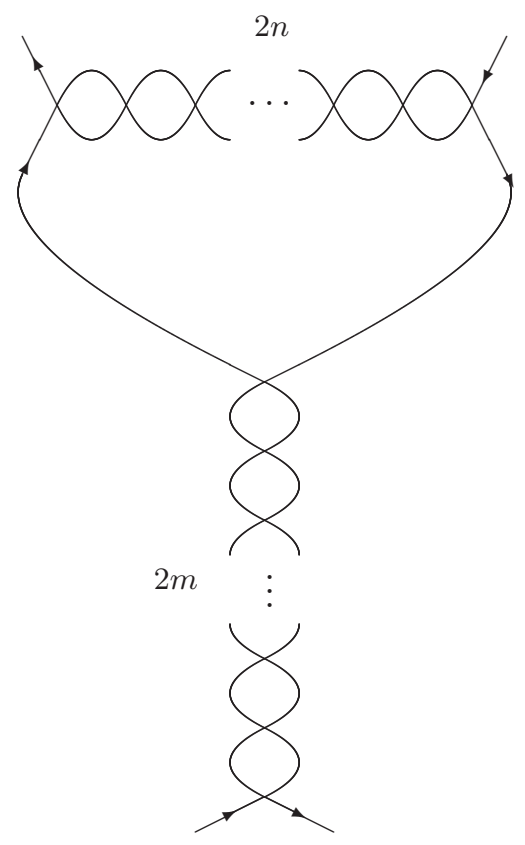

Thus a knowledge of rectangular HOMFLY for the double-braid family can be used to obtain these Racah matrices in rectangular representations. In particular, this provides an alternative derivation for $R=$ [22] — and coincidence with the result of [102] can serve as a check of our factorization hypotheses about the double-braid.

\section{Fundamental representation}

For double braids of above type (two antiparallel braids with even crossing numbers the normalized fundamental HOMFLY are equal to

$$
H_{[1]}^{\{m, n\}}=1+G_{[1]}^{(m, n)}\{A q\}\{A / q\}=1+\frac{\left(A^{2 m}-1\right)\left(A^{2 n}-1\right)}{\left(A^{2}-1\right)\left(A^{-2}-1\right)}\{A q\}\{A / q\}
$$

with $A$-independent factor $F_{[1]}^{(m, n)}$. Thus Alexander polynomial $A l_{[1]}^{(m, n)}=1+m n\{q\}^{2}$ has degree one, and defect [110] of the differential expansion is zero for the entire family. This means that we can use the conjecture of [105] for the shape of differential expansions for rectangular representations of the defect-zero knots. What needs to be found are the $A, q$ dependent coefficients ( $F$-factors), which should be guessed from the limited knowledge of 
HOMFLY for particular members of the family:

\begin{tabular}{|c|ccccccccccc|}
\hline $2 m$ & $\ldots$ & -8 & -6 & -4 & -2 & 0 & 2 & 4 & 6 & 8 & $\cdots$ \\
$2 n$ & & & & & & & & & & & \\
\hline$\cdots$ & & & \multicolumn{1}{c|c}{} \\
8 & & & \multicolumn{1}{c|c}{$10_{1}$} & $U$ & $9_{2}$ & & & & \\
6 & & & & $10_{3}$ & $8_{1}$ & $U$ & $7_{2}$ & $9_{5}$ & & & \\
4 & & & $10_{3}$ & $8_{3}$ & $6_{1}$ & $U$ & $5_{2}$ & $7_{4}$ & $9_{5}$ & & \\
2 & & $10_{1}$ & $8_{1}$ & $6_{1}$ & $4_{1}$ & $U$ & $3_{1}$ & $5_{2}$ & $7_{2}$ & $9_{2}$ & \\
0 & & $U$ & $U$ & $U$ & $U$ & $U$ & $U$ & $U$ & $U$ & $U$ & \\
-2 & & $9_{2}$ & $7_{2}$ & $5_{2}$ & $3_{1}$ & $U$ & $4_{1}$ & $6_{1}$ & $8_{1}$ & $10_{1}$ & \\
-4 & & & $9_{5}$ & $7_{4}$ & $5_{2}$ & $U$ & $6_{1}$ & $8_{3}$ & $10_{3}$ & & \\
-6 & & & & $9_{5}$ & $7_{2}$ & $U$ & $8_{1}$ & $10_{3}$ & & & \\
-8 & & & & & $9_{2}$ & $U$ & $10_{1}$ & & & & \\
$\cdots$ & & & & & & & & & & & \\
\hline
\end{tabular}

$U$ means the unknot (all $F$-factors are zero), the lines/columns with $2 m$ or $2 n= \pm 2$ contain twist knots. The table has obvious symmetries $m \leftrightarrow n$ and $(m, n) \leftrightarrow(-m,-n)$ (in the latter case the knot turns into its mirror, and the change $A, q \longrightarrow A^{-1}, q^{-1}$ should be made in knot polynomial), thus there are not too many different knots in the table. Fortunately, some colored HOMFLY are also available beyond 10 crossings due to powerful family method of $[36,37]$.

For the fundamental representation $R=[1]$ with $d_{[1]}=[N]$ we get from (3.1)

$$
\begin{aligned}
\frac{\sqrt{d_{X} d_{Y}}}{d_{R}} \bar{S}_{X Y} & =\left(\begin{array}{ll}
1 & 0 \\
0 & 1
\end{array}\right)-\frac{\{A q\}\{A / q\}}{\{A\}^{2}}\left(\begin{array}{cc}
1 & -1 \\
-1 & 1
\end{array}\right) \\
& =\frac{1}{[N]^{2}}\left(\begin{array}{cc}
1 & {[N-1][N+1]} \\
{[N-1][N+1]} & -[N-1][N+1]
\end{array}\right)
\end{aligned}
$$

i.e.

$$
\bar{S}_{[1]}=\frac{1}{[N]}\left(\begin{array}{cc}
1 & \sqrt{[N-1][N+1]} \\
\sqrt{[N-1][N+1]} & -1
\end{array}\right)
$$

\section{Twist knots for $R=[22]$}

According to [105], for defect-zero knots $\mathcal{K}^{(0)}$,

$$
\begin{aligned}
H_{[22]}^{\mathcal{K}^{(0)}}= & 1+[2]^{2} Z_{[22]}^{(0)} \cdot F_{[1]}^{\mathcal{K}^{(0)}}(A)+[3] Z_{[22]}^{(0)}\left(Z_{[22]}^{(1)} \cdot F_{[2]}^{\mathcal{K}^{(0)}}(A, q)+Z_{[22]}^{(-1)} \cdot F_{[2]}^{\mathcal{K}^{(0)}}\left(A, q^{-1}\right)\right)+ \\
& +Z_{[22]}^{(1)} Z_{[22]}^{(0)} Z_{[22]}^{(-1)}\left([2]^{2} \mathcal{F}_{3}^{\mathcal{K}^{(0)}}(A, q)+Z_{[22]}^{(0)} \cdot \mathcal{F}_{4}^{\mathcal{K}^{(0)}}(A, q)\right)
\end{aligned}
$$

The two $F$-factors in the first line are known from differential expansions for symmetric representations, the two $\mathcal{F}$-factors in the second line are some $q$-deformations of the cube and the forth power of $F_{[1]}(A)$ - both symmetric under the change $q \leftrightarrow q^{-1}$ (because of the transposition symmetry of $R=[22])$, but very different from, say, $F_{[3]}(A, q=1)$ 
and $F_{[4]}(A, q=1)$. They should, however, satisfy the evolution rule with six eigenvalues $\bar{T}_{[22]}=\operatorname{diag}\left(1,-A, q^{ \pm 2} A^{2},-A^{3}, A^{4}\right)$.

$$
\begin{aligned}
& \mathcal{F}_{3}^{\left[4_{1}\right]}=1 \\
& \mathcal{F}_{3}^{[U]}=0 \\
& \mathcal{F}_{3}^{\left[3_{1}\right]}=-A^{6} \\
& \mathcal{F}_{3}^{\left[5_{2}\right]}=-A^{6}\left(A^{6}+\frac{[6]}{[2]} A^{4}+[3] A^{2}+1\right) \\
& \mathcal{F}_{3}^{\left[7_{2}\right]}=-A^{6}\left(A^{12}+\frac{[6]}{[2]} A^{10}+\frac{[8][6]}{[4][2]} A^{8}+[7] A^{6}+\frac{[4][3]}{[2]} A^{4}+[3] A^{2}+1\right) \\
& \mathcal{F}_{3}^{\left[9_{2}\right]}=-A^{6}\left(A^{18}+\frac{[6]}{[2]} A^{16}+\frac{[8][6]}{[4][2]} A^{14}+\frac{[10][8]}{[4][2]} A^{12}+\frac{[10][6]}{[5]} A^{10}+\frac{[8][3]}{[2]} A^{8}\right. \\
& +\underbrace{\frac{[5][4]}{[2]} A^{6}+\frac{[4][3]}{[2]} A^{4}+[3] A^{2}+1}_{\frac{1}{\left(1-q^{2} A^{2}\right)\left(1-A^{2}\right)\left(1-q^{-2} A^{2}\right)}}) \\
& \text {... } \\
& \text {... } \\
& \mathcal{F}_{4}^{\left[4_{1}\right]}=1 \\
& \mathcal{F}_{4}^{[U]}=0 \\
& \mathcal{F}_{4}^{\left[3_{1}\right]}=A^{8} \\
& \mathcal{F}_{4}^{\left[5_{2}\right]}=A^{8}\left(A^{8}+[2]^{2} A^{6}+\frac{[4][3]}{[2]} A^{4}+[2]^{2} A^{2}+1\right) \\
& \mathcal{F}_{4}^{\left[7_{2}\right]}=A^{8}\left(A^{16}+[2]^{2} A^{14}+\left([3]^{2}+1\right) A^{12}+[4]^{2} A^{10}\right. \\
& \left.+\left(\frac{[6][5]}{[2]}+[3]+1\right) A^{8}+[4]^{2} A^{6}+\left([3]^{2}+1\right) A^{4}+[2]^{2} A^{2}+1\right) \\
& \mathcal{F}_{4}^{\left[9_{2}\right]}=A^{8}\left(A^{24}+[2]^{2} A^{22}+\left([3]^{2}+1\right) A^{20}+\left([4]^{2}+[2]^{2}\right) A^{18}+\left(\frac{[5][4][3]}{[2]}+1\right) A^{16}+\left([6]^{2}+[2]^{2}\right) A^{14}+\right. \\
& +\frac{[4]}{[2]}([11]+[5]+2[3]) A^{12}+\left([6]^{2}+[2]^{2}\right) A^{10}+\left(\frac{[5][4][3]}{[2]}+1\right) A^{8} \\
& +\underbrace{\left([4]^{2}+[2]^{2}\right) A^{6}+\left([3]^{2}+1\right) A^{4}+[2]^{2} A^{2}+1}_{\frac{1}{\left(1-q^{2} A^{2}\right)\left(1-A^{2}\right)^{2}\left(1-q^{-2} A^{2}\right)}})
\end{aligned}
$$

From this we deduce the evolution formulas for twist knots:

$$
\begin{aligned}
F_{1}^{(m)}=A \cdot( & \left.-\frac{A^{2 m}}{\{A\}}+\frac{1}{\{A\}}\right) \\
F_{2}^{(m)}=q A^{2} & \cdot\left(\frac{q^{4 m} A^{4 m}}{\left\{A q^{2}\right\}\{A q\}}-\frac{[2] A^{2 m}}{\left\{A q^{2}\right\}\{A\}}+\frac{1}{\{A q\}\{A\}}\right) \\
\mathcal{F}_{3}^{(m)}=A^{3} \cdot & \left(-\frac{A^{6 m}}{\left\{A q^{2}\right\}\{A\}\left\{A / q^{2}\right\}}+\frac{[3] A^{4 m}}{[2]\left\{A q^{2}\right\}\left\{A / q^{2}\right\}}\left(\frac{q^{4 m}}{\{A q\}}+\frac{q^{-4 m}}{\{A / q\}}\right)\right. \\
& \left.-\frac{[3] A^{2 m}}{\left\{A q^{2}\right\}\{A\}\left\{A / q^{2}\right\}}+\frac{1}{\{A q\}\{A\}\{A / q\}}\right)
\end{aligned}
$$




$$
\begin{aligned}
\mathcal{F}_{4}^{(m)}=A^{4} & \cdot\left(\frac{A^{8 m}}{\{A q\}\{A\}^{2}\{A / q\}}-\frac{[2]^{2} A^{6 m}}{\left\{A q^{2}\right\}\{A\}^{2}\left\{A / q^{2}\right\}}+\frac{[3] A^{4 m}\left(q^{4 m}+q^{-4 m}\right)}{\left\{A q^{2}\right\}\{A q\}\{A / q\}\left\{A / q^{2}\right\}}\right. \\
& \left.-\frac{[2]^{2} A^{2 m}}{\left\{A q^{2}\right\}\{A\}^{2}\left\{A / q^{2}\right\}}+\frac{1}{\{A q\}\{A\}^{2}\{A / q\}}\right)
\end{aligned}
$$

while for symmetric representations we have [107] (note that eigenvalues are now different, $\left.(-)^{i} A^{i} q^{i(i-1)}\right)$ :

$$
\begin{aligned}
F_{3}^{(m)}=q^{3} A^{3} \cdot & \left(-\frac{q^{12 m} A^{6 m}}{\left\{A q^{4}\right\}\left\{A q^{3}\right\}\left\{A q^{2}\right\}}+\frac{[3] q^{4 m} A^{4 m}}{\left\{A q^{4}\right\}\left\{A q^{2}\right\}\{A q\}}-\frac{[3] A^{2 m}}{\left\{A q^{3}\right\}\left\{A q^{2}\right\}\{A\}}+\frac{1}{\left\{A q^{2}\right\}\{A q\}\{A\}}\right) \\
F_{4}^{(m)}=q^{6} A^{4} \cdot & \left(\frac{q^{24 m} A^{8 m}}{\left\{A q^{6}\right\}\left\{A q^{5}\right\}\left\{A q^{4}\right\}\left\{A q^{3}\right\}}-\frac{[4] q^{12 m} A^{6 m}}{\left\{A q^{6}\right\}\left\{A q^{4}\right\}\left\{A q^{3}\right\}\left\{A q^{2}\right\}}+\frac{\frac{[4][3]}{[2]} q^{4 m} A^{4 m}}{\left\{A q^{5}\right\}\left\{A q^{4}\right\}\left\{A q^{2}\right\}\{A q\}}-\right. \\
& \left.-\frac{[4] A^{2 m}}{\left\{A q^{4}\right\}\left\{A q^{3}\right\}\left\{A q^{2}\right\}\{A\}}+\frac{1}{\left\{A q^{3}\right\}\left\{A q^{2}\right\}\{A q\}\{A\}}\right)
\end{aligned}
$$

Of course, these formulas can be checked for other low-intersection twist knots $6_{1}, 8_{1}, 10_{1}$, respectively for $m=-2,-3,-4$.

\section{Factorization of differential expansion for double-braids}

Much more interesting are the non-twist knots from the double-braid family. Already in the first two examples we obtain:

$$
\begin{aligned}
H_{[22]}^{8_{3}}= & 1-[2]^{2} A^{-2} Z_{[22]}^{(0)} F_{1}^{(2)}(A) F_{1}^{(-2)}(A)+[3] Z_{[22]}^{(0)}\left(G_{[2]}^{8_{3}}(A, q) Z_{[22]}^{(1)}+G_{[2]}^{8_{3}}\left(A, q^{-1}\right) Z_{[22]}^{(-1)}\right)- \\
& -A^{-6}[2]^{2} Z_{[22]}^{(1)} Z_{[22]}^{(0)} Z_{[22]}^{(-1)} \mathcal{F}_{3}^{(2)} \mathcal{F}_{3}^{(-2)}+A^{-8} Z_{[22]}^{(1)}\left(Z_{[22]}^{(0)}\right)^{2} Z_{[22]}^{(-1)} \mathcal{F}_{4}^{(2)} \mathcal{F}_{4}^{(-2)} \\
H_{[22]}^{10_{3}}= & 1-[2]^{2} A^{-2} Z_{[22]}^{(0)} F_{1}^{(2)}(A) F_{1}^{(-3)}(A)+[3] Z_{[22]}^{(0)}\left(G_{[2]}^{10_{3}}(A, q) Z_{[22]}^{(1)}+G_{[2]}^{10_{3}}\left(A, q^{-1}\right) Z_{[22]}^{(-1)}\right)- \\
& -A^{-6}[2]^{2} Z_{[22]}^{(1)} Z_{[22]}^{(0)} Z_{[22]}^{(-1)} \mathcal{F}_{3}^{(2)} \mathcal{F}_{3}^{(-3)}+A^{-8} Z_{[22]}^{(1)}\left(Z_{[22]}^{(0)}\right)^{2} Z_{[22]}^{(-1)} \mathcal{F}_{4}^{(2)} \mathcal{F}_{4}^{(-3)}
\end{aligned}
$$

with $G_{[2]}^{8_{3}}=q^{-2} A^{-4} F_{2}^{(2)} F_{2}^{(-2)}$ and $G_{[2]}^{10_{3}}=q^{-2} A^{-4} F_{2}^{(2)} F_{2}^{(-3)}$.

Clearly, the coefficients $\mathcal{F}$ factorize into products of the twist-family $\mathcal{F}$-factors!

Assuming that this amusing factorization is always true for all double braids, we conjecture:

$$
\begin{aligned}
H_{[22]}^{(m, n)}= & 1-[2]^{2} A^{-2} Z_{[22]}^{(0)} \cdot F_{1}^{(m)}(A) F_{1}^{(n)}(A)+ \\
& +[3] A^{-4} Z_{[22]}^{(0)}\left(q^{-2} Z_{[22]}^{(1)} \cdot F_{2}^{(m)}(A, q) F_{2}^{(n)}(A, q)+q^{2} Z_{[22]}^{(-1)} \cdot F_{2}^{(m)}\left(A, q^{-1}\right) F_{2}^{(n)}\left(A, q^{-1}\right)\right)- \\
& -A^{-6}[2]^{2} Z_{[22]}^{(1)} Z_{[22]}^{(0)} Z_{[22]}^{(-1)} \cdot \mathcal{F}_{3}^{(m)} \mathcal{F}_{3}^{(n)}+A^{-8} Z_{[22]}^{(1)}\left(Z_{[22]}^{(0)}\right)^{2} Z_{[22]}^{(-1)} \cdot \mathcal{F}_{4}^{(m)} \mathcal{F}_{4}^{(n)}
\end{aligned}
$$

what can be tested in the other two available examples in table $(3.2)-7_{4}$ and $9_{5}$. This conjecture provides a double-evolution matrix, which through (2.2) gives the entries of $\sqrt{d_{\bar{X}} d_{\bar{Y}}} \bar{S}_{\bar{X} \bar{Y}}$. This actually proves the conjecture, because the result reproduces the matrix $\bar{S}$, derived from a very different double evolution in [102]: 


$$
\bar{S}^{[2,2]}=\frac{1}{d_{[2,2]}}\left(\begin{array}{cccccc}
\sqrt{\bar{d}_{1}} & \sqrt{\bar{d}_{2}} & \sqrt{\bar{d}_{3}} & \sqrt{\bar{d}_{4}} & \sqrt{\bar{d}_{5}} & \sqrt{\bar{d}_{6}} \\
12 & \frac{D_{1} D_{-1}}{[2]^{2} D_{2} D_{-2}} \gamma_{1}-\frac{\sqrt{D_{3} D_{1} D_{-1} D_{0}}}{[2]^{2} D_{2} D_{-2}} \gamma_{2} & \frac{\sqrt{D_{-3} D_{-1}} D_{0} D_{1}}{[2]^{2} D_{2} D_{-2}} \gamma_{3} & -\frac{\sqrt{D_{3} D_{1} D_{-1} D_{-3} D_{1} D_{-1}}}{[2]^{2}[3] D_{2} D_{-2}} \gamma_{4}-15 \\
13 & 23 & \frac{D_{0}^{2}}{[2]^{2}[3] D_{2} D_{-2}} \gamma_{5} & -\frac{\sqrt{D_{3} D_{1} D_{-1} D_{-3} D_{0}^{2}[3]}}{[2]^{2} D_{2} D_{-2}} & -24 & 14 \\
14 & 24 & 34 & 33 & -23 & 13 \\
15 & 25 & -24 & -23 & 22 & -12 \\
16 & -15 & 14 & 13 & -12 & 11
\end{array}\right)
$$

with $\gamma_{1}=[3] D_{2} D_{-2}-[2]^{2}, \quad \gamma_{2}=D_{2} D_{-3}-[2], \gamma_{3}=D_{3} D_{-2}-[2], \gamma_{4}=D_{3} D_{-3}-2[3]-1, \gamma_{5}=$ $D_{3} D_{2} D_{-2} D_{-3}-D_{3} D_{-3}+[2]^{2}$.

Here $D_{i}=\frac{\left\{A q^{i}\right\}}{\{q\}}$, while $\bar{d}_{i}$ are quantum dimensions of the six irreducible representations in the product $[22] \otimes \overline{[22]}$ :

$$
\begin{array}{lll}
\bar{d}_{1}=1, & \bar{d}_{2}=D_{1} D_{-1}, & \bar{d}_{3}=\frac{D_{3} D_{0}^{2} D_{-1}}{[2]^{2}}, \\
\bar{d}_{4}=\frac{D_{1} D_{0}^{2} D_{-3}}{[2]^{2}}, & \bar{d}_{5}=\frac{D_{3} D_{1}^{2} D_{-1}^{2} D_{-3}}{[3]^{2}}, & \bar{d}_{6}=\frac{D_{3} D_{2}^{2} D_{1} D_{-1} D_{-2}^{2} D_{-3}}{[3]^{2}[2]^{4}}
\end{array}
$$

The matrix has two symmetries: $\bar{S}_{i, j}=\bar{S}_{j, i}$ and $\bar{S}_{i, j}= \pm \bar{S}_{7-j, 7-i}$, the signs are shown explicitly in above table, where "12" at the place of $\bar{S}_{21}$ means that this element is equal to $\bar{S}_{12}$ etc. Presented is the matrix, obtained by our new procedure: it differs a little (in signs and order of lines - as allowed by conjugation freedom) from the one in [102].

As already mentioned, another inclusive Racah matrix $S$ is obtained from (2.1), by diagonalization of $\bar{T} \bar{S} \bar{T}$ with

$$
\bar{T}=\operatorname{diag}\left(1,-A, q^{2} A^{2}, q^{-2} A^{2},-A^{3}, A^{4}\right)
$$

The eigenvalues are equal to

$$
T^{-1}=A^{4} \cdot \operatorname{diag}\left(q^{8},-q^{4}, q^{2}, 1 / q^{2},-1 / q^{4}, 1 / q^{8}\right)
$$

and the entries of $S_{X Y}$ are then given by Cramer rule as minors of the matrix $\bar{T} \bar{S} \bar{T}-T$, like in a similar procedure, described in [102].

Factorization property holds for symmetric and antisymmetric representations as well: for typical examples

$$
\begin{aligned}
H_{[3]}^{(m, n)}= & 1-[3] A^{-2} Z_{[4]}^{(0)} \cdot F_{1}^{(m)}(A) F_{1}^{(n)}(A)+ \\
& +[3] q^{-2} A^{-4} Z_{[3]}^{(1)} Z_{[4]}^{(0)} \cdot F_{2}^{(m)}(A, q) F_{2}^{(n)}(A, q)-q^{-6} A^{-6} Z_{[4]}^{(2)} Z_{[4]}^{(1)} Z_{[4]}^{(0)} \cdot F_{3}^{(m)}(A, q) F_{3}^{(n)}(A, q)
\end{aligned}
$$

and

$$
H_{[1111]}^{(m, n)}=1-[4] A^{-2} Z_{[1111]}^{(0)} \cdot F_{1}^{(m)}(A) F_{1}^{(n)}(A)+\frac{[4][3]}{[2]} q^{2} A^{-4} Z_{[1111]}^{(0)} Z_{[1111]}^{(-1)} \cdot F_{2}^{(m)}\left(A, q^{-1}\right) F_{2}^{(n)}\left(A, q^{-1}\right)-
$$




$$
\begin{aligned}
& -[4] q^{6} A^{-6} Z_{[1111]}^{(0)} Z_{[1111]}^{(-1)} Z_{[1111]}^{(-2)} \cdot F_{3}^{(m)}\left(A, q^{-1}\right) F_{3}^{(n)}\left(A, q^{-1}\right) \\
& +q^{12} A^{-8} Z_{[1111]}^{(0)} Z_{[1111]}^{(-1)} Z_{[1111]}^{(-2)} Z_{[1111]}^{(-3)} \cdot F_{4}^{(m)}\left(A, q^{-1}\right) F_{4}^{(n)}\left(A, q^{-1}\right)
\end{aligned}
$$

However, factorization is not extended to polynomials of the form

$$
H_{R}^{\left(m_{1}, \ldots, m_{k}\right)}=d_{R} \cdot\left(\bar{S} \bar{T}^{2 m_{1}} \bar{S} \bar{T}^{2 m_{2}} \bar{S} \ldots \bar{S} \bar{T}^{2 m_{k}} \bar{S}\right)_{\emptyset \emptyset}
$$

with $k \neq 2$ - it fails already for the fundamental representations of the simplest knots of thus type, like $5_{1}=(1,1,1,1)$ and $7_{3}=(1,1,1,2)$.

Instead we claim that it is extended to other rectangular representations - but only for double antiparallel braids. This is, however, exactly what we need to get the matrices $\bar{S}$.

\section{Other rectangular representations $R=\left[r^{s}\right]$}

Now the plan is clear.

- We take general differential expansion of rectangular HOMFLY for defect-zero knots from [105]. It has the form

$$
H_{\left[r^{s}\right]}^{\mathcal{K}^{(0)}}=\sum_{\mathcal{A}, \mathcal{B}} C_{\left[r^{s}\right]}^{\mathcal{A}, \mathcal{B}} \cdot Z_{\mathcal{A}, \mathcal{B}} \cdot \mathcal{F}_{\mathcal{A}, \mathcal{B}}^{\mathcal{K}^{(0)}}
$$

where sum goes over peculiar multi-floor pyramids, labeled by pairs of the Young-like diagrams $\mathcal{A}, \mathcal{B}$, All the coefficients $\mathcal{F}_{\mathcal{A}, \mathcal{B}}^{4_{1}}=1$ for the figure-eight knot and $\mathcal{F}_{\mathcal{A}, \mathcal{B}}^{3_{1}}$ are just monomials (powers of $q$ and $A$ ) for the trefoil.

- Guess a general formula for the coefficients $\mathcal{F}_{\mathcal{A}, \mathcal{B}}^{(m)}$ in the case of twist knots.

- Assume that antiparallel double braids satisfy factorization

$$
\mathcal{F}_{\mathcal{A}, \mathcal{B}}^{(m, n)}=\frac{\mathcal{F}_{\mathcal{A}, \mathcal{B}}^{(m)} \mathcal{F}_{\mathcal{A}, \mathcal{B}}^{(n)}}{\mathcal{F}_{\mathcal{A}, \mathcal{B}}^{(1)} \mathcal{F}_{\mathcal{A}, \mathcal{B}}^{(-1)}}
$$

where $\mathcal{F}_{\mathcal{A}, \mathcal{B}}^{(1)}=\left(-q^{|\mathcal{A}|-|\mathcal{B}|} A^{2}\right)^{|\mathcal{A}, \mathcal{B}|}$ and $\mathcal{F}_{\mathcal{A}, \mathcal{B}}^{(-1)}=1$

- Representing double-braid case $(k=2)$ in the form of a double evolution in $m_{1}$ and $m_{2}$, use (2.2) to extract the Racah matrix $\bar{S}_{\left[r^{s}\right]}$.

- Diagonalizing $\bar{T} \bar{S} \bar{T}$ with the help of known eigenvalues and Cramer rule, as explained in [102], obtain another Racah matrix $S_{\left[r^{s}\right]}$.

- Calculate $H_{\left[r^{s}\right]}$ for any desired arborescent knot by the technique of $[36,37,94]$ (some additional care/checks can be needed with effective vertices in the multi-finger case) and make possible checks.

The most artful part for today is the second step: revealing the structure of $\mathcal{F}$ for twist knots from very special examples - symmetric and antisymmetric representations $R=[r]$ and $R=\left[1^{r}\right]$ and the only double-floor example, provided by $R=[22]$. 
More formally, complementing the conjecture of [105] by above suggestions, we look for the $\left[r^{s}\right]$-colored HOMFLY in the following form:

$$
\begin{aligned}
& H_{\left[r^{s}\right]}^{(m, n)} \stackrel{?}{=} \sum_{F=0}^{\min (r, s)} \sum_{\{\mathcal{A}, \mathcal{B}\}} W_{\mathcal{A}, \mathcal{B}} \cdot \frac{\mathcal{F}_{\mathcal{A}, \mathcal{B}}^{(m)} \mathcal{F}_{\mathcal{A}, \mathcal{B}}^{(n)}}{\mathcal{F}_{\mathcal{A}, \mathcal{B}}^{(1)} \mathcal{F}_{\mathcal{A}, \mathcal{B}}^{(-1)}} \cdot\left(\prod_{f=1}^{F}\left(C_{a_{f}+b_{f}}^{b_{f}}\right)^{2} C_{r+b_{f}}^{p_{f}} C_{s+a_{f}}^{p_{f}}\right) \\
& a_{F} \ldots 0 \ldots-b_{F}
\end{aligned}
$$

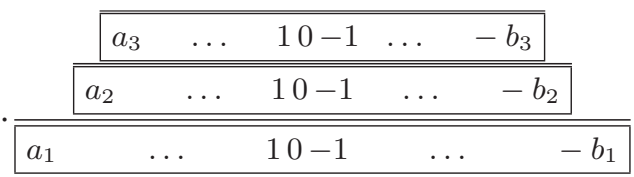

$$
\begin{aligned}
& =1+\sum_{F=1}^{\min (r, s)} \sum_{\substack{0 \leq a_{F}<\ldots<a_{3}<a_{2}<a_{1}<r \\
0 \leq b_{F}<\ldots<b_{3}<b_{2}<b_{1}<s}} \prod_{f^{\prime}<f^{\prime \prime}}^{F}\left(\frac{\left[a_{f^{\prime}}-a_{f^{\prime \prime}}\right]\left[b_{f^{\prime}}-b_{f^{\prime \prime}}\right]}{\left[a_{f^{\prime}}+b_{f^{\prime \prime}}+1\right]\left[a_{f^{\prime \prime}}+b_{f^{\prime}}+1\right]}\right)^{2} \cdot \frac{\mathcal{F}_{\mathcal{A}, \mathcal{B}}^{(m)} \mathcal{F}_{\mathcal{A}, \mathcal{B}}^{(n)}}{\mathcal{F}_{\mathcal{A}, \mathcal{B}}^{(1)} \mathcal{F}_{\mathcal{A}, \mathcal{B}}^{(-1)}} . \\
& \cdot \prod_{f=1}^{F}\left(\left(\frac{\left[a_{f}+b_{f}\right] !}{\left(\left[a_{f}\right] !\left[b_{f}\right] !\right.}\right)^{2} \frac{\left[r+b_{f}\right] !\left[s+a_{f}\right] !}{\left[r-1-a_{f}\right] !\left[s-1-b_{f}\right] !\left(\left[a_{f}+b_{f}+1\right] !\right)^{2}} \prod_{i_{f}=-b_{f}}^{a_{f}}\left\{A q^{i_{f}+r}\right\}\left\{A q^{i_{f}-s}\right\}\right)
\end{aligned}
$$

and our new goal is to associate the evolution functions $\mathcal{F}_{\mathcal{A}, \mathcal{B}}^{(m)}$ with every non-empty pyramid

$$
\{\mathcal{A}, \mathcal{B}\}=\left\{r>a_{1}>a_{2} \ldots>a_{f} \geq 0\right\} \cup\left\{s>b_{1}>b_{2}>\ldots>b_{f} \geq 0\right\}
$$

These functions depend on the eigenvalues $\lambda_{c}$, which are powers of $A$ and $q$. However, for concrete pyramid $\{\mathcal{A}, \mathcal{B}\}$ the function $\mathcal{F}_{\mathcal{A}, \mathcal{B}}^{(m)}$ depends on only some of these eigenvalues and this subset depends on the pyramid and not on $r$ and $s$ (these parameters, however, select particular pyramides that contribute to $\left.H_{\left[r^{s}\right]}\right)$. Schematically,

$$
\mathcal{F}_{\mathcal{A}, \mathcal{B}}^{(m)}=\sum_{c \in I_{\mathcal{A}, \mathcal{B}}} \lambda_{c}^{2 m} \cdot \frac{\xi_{c}}{\prod_{i \in J_{\mathcal{A}, \mathcal{B}}}\left\{A q^{i}\right\}}
$$

The first two tasks are to describe the sets $I_{\mathcal{A}, \mathcal{B}}$ and $J_{\mathcal{A}, \mathcal{B}}$, and the third is the finding of the combinatorial coefficients $\xi_{c}$. In this paper they are fulfilled only partly — for the single-floor diagrams $\{\mathcal{A}, \mathcal{B}\}$.

\section{On the structure of $\mathcal{F}_{\mathcal{A}, \mathcal{B}}^{(m)}$}

\subsection{Already known cases}

To begin with let us remind this structure in the case of symmetric representations $[r]$, where the relevant diagrams are just single-floor boxes $a \ldots 0$ with $b=0$. Then, from [107] we know the answer:

$$
\mathcal{F}_{a \ldots 0}^{(m)}=q^{\frac{a(a+1)}{2}} A^{a+1} \sum_{c=0}^{a+1} \frac{(-)^{c}[a+1] !}{[c] ![a+1-c] !} \cdot \lambda_{c}^{2 m} \cdot \frac{\left\{A q^{2 c-1}\right\}}{\prod_{i=c-1}^{c+a}\left\{A q^{i}\right\}}
$$


It depends on $a+2$ eigenvalues (of the quantum $\mathcal{R}$-matrix) $\lambda_{c}=(-)^{c} q^{c(c-1)} A^{c}$. Likewise, for the antisymmetric representations $\left[1^{s}\right]$ contributing are only the boxes $0 \ldots-b$ with $a=0$, and

$$
\mathcal{F}_{0 \ldots-b}^{(m)}=q^{-\frac{b(b+1)}{2}} A^{b+1} \sum_{c=0}^{a+1} \sum_{c=0}^{b+1} \frac{(-)^{c}[b+1] !}{[c] ![b+1-c] !} \cdot \lambda_{-c}^{2 m} \cdot \frac{\left\{A q^{1-2 c}\right\}}{\prod_{i=c-1}^{c+b}\left\{A q^{-i}\right\}}
$$

with $b+2$ eigenvalues $\lambda_{-c}=(-)^{c} q^{-c(c-1)} A^{c}$. In fact, it is already a challenge to unify (7.1) and (7.2) into a single formula.

The knowledge of the $R=[22]$ case adds to

$$
\begin{aligned}
\mathcal{F}_{0}^{(m)} & =A \cdot\left(\lambda_{0}^{2 m} \frac{\{A / q\}}{\{A / q\}\{A\}}-\lambda_{1}^{2 m} \frac{\{A q\}}{\{A\}\{A q\}}\right)=A \cdot \frac{\lambda_{0}^{2 m}-\lambda_{1}^{2 m}}{\{A\}}=\frac{1-A^{2 m}}{1-A^{-2}} \\
\mathcal{F}_{10}^{(m)} & =q A^{2} \cdot\left(\lambda_{0}^{2 m} \frac{\{A / q\}}{\{A / q\}\{A\}\{A q\}}-[2] \lambda_{1}^{2 m} \frac{\{A q\}}{\{A\}\{A q\}\left\{A q^{2}\right\}}+\lambda_{2}^{2 m} \frac{\left\{A q^{3}\right\}}{\{A q\}\left\{A q^{2}\right\}\left\{A q^{3}\right\}}\right) \\
\mathcal{F}_{0-1}^{(m)} & =q^{-1} A^{2} \cdot\left(\lambda_{0}^{2 m} \frac{\{A q\}}{\{A q\}\{A\}\{A / q\}}-[2] \lambda_{1}^{2 m} \frac{\{A / q\}}{\{A\}\{A / q\}\left\{A / q^{2}\right\}}+\lambda_{-2}^{2 m} \frac{\left\{A / q^{3}\right\}}{\{A / q\}\left\{A / q^{2}\right\}\left\{A / q^{3}\right\}}\right)
\end{aligned}
$$

two more pyramids:

$$
\begin{aligned}
\mathcal{F}^{(m)}=A^{3} \cdot & \left(\frac{\lambda_{0}^{2 m}}{\{A q\}\{A\}\{A / q\}}-\frac{[3] \lambda_{1}^{2 m}}{\left\{A q^{2}\right\}\{A\}\left\{A / q^{2}\right\}}+\frac{[3] \lambda_{2}^{2 m}}{[2]\left\{A q^{2}\right\}\{A q\}\left\{A / q^{2}\right\}}\right. \\
& \left.+\frac{[3] \lambda_{-2}^{2 m}}{[2]\left\{A q^{2}\right\}\{A / q\}\left\{A / q^{2}\right\}}-\frac{\lambda_{03}^{2 m}}{\left\{A q^{2}\right\}\{A\}\left\{A / q^{2}\right\}}\right) \\
\mathcal{F}_{0}^{(m)}=A^{4} \cdot & \left(\frac{\lambda_{0}^{2 m}}{\{A q\}\{A\}^{2}\{A / q\}}-\frac{[2]^{2} \lambda_{1}^{2 m}}{\left\{A q^{2}\right\}\{A\}^{2}\left\{A / q^{2}\right\}}+\frac{[3] \lambda_{2}^{2 m}}{\left\{A q^{2}\right\}\{A q\}\{A / q\}\left\{A / q^{2}\right\}}\right. \\
& \left.+\frac{[3] \lambda_{-2}^{2 m}}{\left\{A q^{2}\right\}\{A q\}\{A / q\}\left\{A / q^{2}\right\}}-\frac{[2]^{2} \lambda_{03}^{2 m}}{\left\{A q^{2}\right\}\{A\}^{2}\left\{A / q^{2}\right\}}+\frac{\lambda_{04}^{2 m}}{\{A q\}\{A\}^{2}\{A / q\}}\right)
\end{aligned}
$$

with five and six eigenvalues, associated with the six irreducible representations in the product $[22] \otimes \overline{[22]}$ :

$$
\lambda_{0}=1, \quad \lambda_{1}=-A=\lambda_{-1}, \quad \lambda_{ \pm 2}=q^{ \pm 2} A^{2}, \quad \lambda_{03}=-A^{3}, \quad \lambda_{04}=A^{4}
$$

\subsection{The first unknown case: $R=[33]$}

Now there are ten irreps in $[33] \otimes \overline{[33]}$ and ten eigenvalues: six "old" ones, the same as (7.5), and four "new":

$$
\lambda_{+3}=-q^{6} A^{3}, \quad \lambda_{+_{0} 4}=q^{4} A^{4}, \quad \lambda_{+_{0} 5}=-q^{4} A^{5}, \quad \lambda_{+_{0} 6}=q^{6} A^{6}
$$

(somewhat strange labels in $\lambda_{{ }_{0} c}$ emphasize that the powers of $q$ are smaller than $c(c-1)$ in $\lambda_{+c}$, familiar from symmetric representation case (7.1), see section 7.4 below for a more systematic description). The "old" pyramids are described by the same formulas (7.3) and (7.4), involving only "old" eigenvalues (7.5). Of the four "new" pyramids one is still described by (7.1):

$$
\begin{aligned}
\mathcal{F}_{210}^{(m)}=q^{3} A^{3} & \cdot\left(\lambda_{0}^{2 m} \frac{\{A / q\}}{\{A / q\}\{A\}\{A q\}\left\{A q^{2}\right\}}-[3] \lambda_{1}^{2 m} \frac{\{A q\}}{\{A\}\{A q\}\left\{A q^{2}\right\}\left\{A q^{3}\right\}}+\right. \\
& \left.+[3] \lambda_{2}^{2 m} \frac{\left\{A q^{3}\right\}}{\{A q\}\left\{A q^{2}\right\}\left\{A q^{3}\right\}\left\{A q^{4}\right\}}-\lambda_{3}^{2 m} \frac{\left\{A q^{5}\right\}}{\left\{A q^{2}\right\}\left\{A q^{3}\right\}\left\{A q^{4}\right\}\left\{A q^{5}\right\}}\right)
\end{aligned}
$$


while three more we should guess:

$$
\begin{aligned}
& \mathcal{F}_{2210-1}^{(m)} \stackrel{?}{=} q^{2} A^{4} \cdot\left(\frac{\lambda_{0}^{2 m}}{\left\{A q^{2}\right\}\{A q\}\{A\}\{A / q\}}-\frac{[4] \lambda_{1}^{2 m}}{\left\{A q^{3}\right\}\left\{A q^{2}\right\}\{A\}\left\{A / q^{2}\right\}}++\frac{[4] \lambda_{2}^{2 m}}{\left\{A q^{4}\right\}\left\{A q^{2}\right\}\{A q\}\left\{A / q^{2}\right\}}\right. \\
& +\frac{\frac{[4]}{[2]} \lambda_{-2}^{2 m}}{\left\{A q^{3}\right\}\left\{A q^{2}\right\}\{A / q\}\left\{A / q^{2}\right\}}-\frac{\frac{[4]}{[3]} \lambda_{+3}^{2 m}}{\left\{A q^{4}\right\}\left\{A q^{3}\right\}\left\{A q^{2}\right\}\left\{A / q^{2}\right\}}-\frac{\frac{[4][2]}{[3]} \lambda_{03}^{2 m}}{\left\{A q^{4}\right\}\left\{A q^{2}\right\}\{A\}\left\{A / q^{2}\right\}}+ \\
& \left.+\frac{\lambda_{+0^{4}}^{2 m}}{\left\{A q^{4}\right\}\left\{A q^{3}\right\}\{A q\}\left\{A / q^{2}\right\}}\right)
\end{aligned}
$$

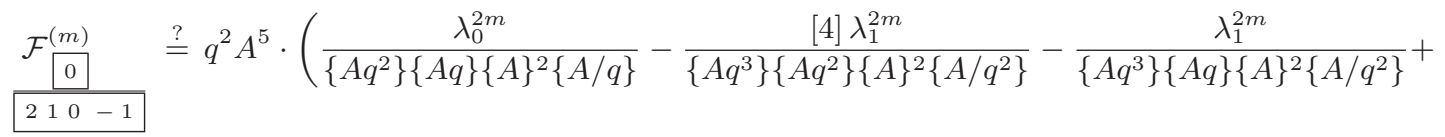

$$
\begin{aligned}
& +\frac{\frac{[4][3]}{[2]} \lambda_{2}^{2 m}}{\left\{A q^{4}\right\}\left\{A q^{2}\right\}\{A q\}\{A / q\}\left\{A / q^{2}\right\}}+\frac{[4] \lambda_{-2}^{2 m}}{\left\{A q^{3}\right\}\left\{A q^{2}\right\}\{A q\}\{A / q\}\left\{A / q^{2}\right\}}- \\
& -\frac{\frac{[4]}{[2]} \lambda_{+3}^{2 m}}{\left\{A q^{4}\right\}\left\{A q^{3}\right\}\left\{A q^{2}\right\}\{A / q\}\left\{A / q^{2}\right\}}-\frac{[4][2] \lambda_{03}^{2 m}}{\left\{A q^{4}\right\}\left\{A q^{2}\right\}\{A\}^{2}\left\{A / q^{2}\right\}}+ \\
& +\frac{[3] \lambda_{+04}^{2 m}}{\left\{A q^{4}\right\}\left\{A q^{3}\right\}\{A q\}\{A\}\left\{A / q^{2}\right\}}+\frac{\frac{[4]}{[2]} \lambda_{04}^{2 m}}{\left\{A q^{4}\right\}\{A q\}\{A\}^{2}\{A / q\}} \\
& \left.-\frac{\lambda_{+0^{5}}^{2 m}}{\left\{A q^{4}\right\}\left\{A q^{2}\right\}\{A q\}\{A\}\{A / q\}}\right)
\end{aligned}
$$

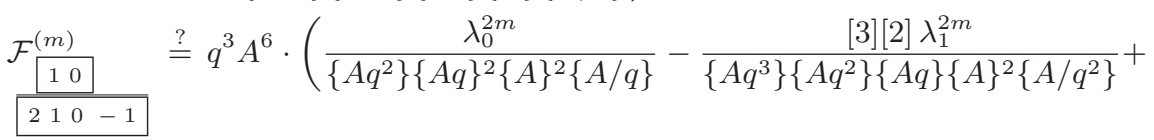

$$
\begin{aligned}
& +\frac{[3]^{2} \lambda_{2}^{2 m}}{\left\{A q^{4}\right\}\left\{A q^{2}\right\}\{A q\}^{2}\{A / q\}\left\{A / q^{2}\right\}}+\frac{\frac{[4][3]}{[2]} \lambda_{-2}^{2 m}}{\left\{A q^{3}\right\}\left\{A q^{2}\right\}^{2}\{A q\}\{A / q\}\left\{A / q^{2}\right\}}- \\
& -\frac{[4] \lambda_{+3}^{2 m}}{\left\{A q^{4}\right\}\left\{A q^{3}\right\}\left\{A q^{2}\right\}\{A\}\{A / q\}\left\{A / q^{2}\right\}}-\frac{[4][2]^{2} \lambda_{03}^{2 m}}{\left\{A q^{4}\right\}\left\{A q^{2}\right\}^{2}\{A\}^{2}\left\{A / q^{2}\right\}}+ \\
& +\frac{[3]^{2} \lambda_{+04}^{2 m}}{\left\{A q^{4}\right\}\left\{A q^{3}\right\}\{A q\}^{2}\{A\}\left\{A / q^{2}\right\}}+\frac{\frac{[4][3]}{[2]} \lambda_{04}^{2 m}}{\left\{A q^{4}\right\}\left\{A q^{3}\right\}\{A q\}\{A\}^{2}\{A / q\}}- \\
& \left.-\frac{[3][2] \lambda_{+0^{5}}^{2 m}}{\left\{A q^{4}\right\}\left\{A q^{2}\right\}^{2}\{A q\}\{A\}\{A / q\}}+\frac{\lambda_{+06}^{2 m}}{\left\{A q^{3}\right\}\left\{A q^{2}\right\}^{2}\{A q\}^{2}\{A\}}\right)
\end{aligned}
$$

Such guesses are motivated by a number of requirements:

- similarity to (7.1), (7.2) and (7.4), implying that $\mathcal{F}_{\mathcal{A}, \mathcal{B}}$ is a sum of powers $\lambda_{c}^{2 m}$ with nicely factorized coefficients,

- observation in these examples of certain regularity in the positions of poles, coming from $\left\{A q^{i}\right\}$ with $-2 b<i<2 a$ in denominators, for terms with different $\lambda_{c}^{2 m}$,

- requirement that each term in the sum over $\lambda_{c}$ depends on even powers $q$,

- requirement, that entire $\mathcal{F}_{\mathcal{A}, \mathcal{B}}^{(m)}$ is a polynomial for any $m$, i.e. all poles cancel after summation over $c$,

- vanishing $\mathcal{F}_{\mathcal{A}, \mathcal{B}}^{(0)}=0$ at $m=0$, i.e. for the unknot,

- $\mathcal{F}_{\mathcal{A}, \mathcal{B}}^{(-1)}=1$ for $m=-1$, i.e. for the figure eight knot, see [105], 
- $\mathcal{F}_{\mathcal{A}, \mathcal{B}}^{(1)}=\prod_{f}\left(-q^{a_{f}-b_{f}} A^{2}\right)^{a_{f}+b_{f}+1}$ for $m=1$, i.e. for the trefoil, see [105].

For the particular case of $R=$ [33] these are rather restrictive requirements and the above guess is actually less ambiguous, than it can seem. In any case, the real confirmation comes a posteriori - from the final expressions for Racah matrices and reasonable answers they provide for the [33]-colored knot polynomials.

With these guesses we can calculate

$$
\begin{aligned}
& H_{[33]}^{(m, n)} \stackrel{?}{=} 1-[3][2] Z_{[33]}^{(0)} \cdot A^{-2} \mathcal{F}_{0}^{(m)} \mathcal{F}_{0}^{(n)}+ \\
& +\left([3]^{2} Z_{[33]}^{(+1)} \cdot q^{-2} A^{-4} \mathcal{F}_{10}^{(m)} \mathcal{F}_{10}^{(n)}+\frac{[3][4]}{[2]} Z_{[33]}^{(-1)} \cdot q^{2} A^{-4} \mathcal{F}_{0-1}^{(m)} \mathcal{F}_{0-1}^{(n)}\right) Z_{[33]}^{(0)}- \\
& -\left([4] Z_{[33]}^{(+2)} \cdot q^{-6} A^{-6} \mathcal{F}_{\frac{210}{(m)}}^{\mathcal{F}_{210}^{(n)}}+[4][2]^{2} Z_{[33]}^{(-1)} \cdot A^{-6} \mathcal{F}_{10-1}^{(m)} \mathcal{F}_{10-1}^{(n)}\right) Z_{[33]}^{(+1)} Z_{[33]}^{(0)}+ \\
& +\left([3]^{2} Z_{[33]}^{(+2)} \cdot q^{-4} A^{-8} \mathcal{F}_{[210-1}^{(m)} \mathcal{F}_{[210-1}^{(n)}+\frac{[3][4]}{[2]} Z_{[33]}^{(0)} \cdot A^{-8} \mathcal{F}_{[0]}^{(m)} \mathcal{F}_{[0]}^{(n)}\right) Z_{[33]}^{(+1)} Z_{[33]}^{(0)} Z_{[33]}^{(-1)}-
\end{aligned}
$$

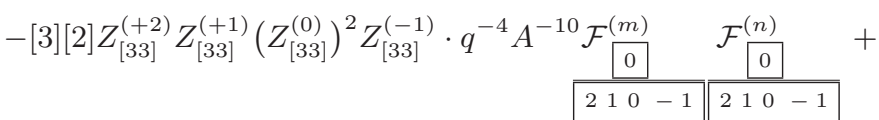

$$
\begin{aligned}
& \begin{array}{rl}
+Z_{[33]}^{(+2)}\left(Z_{[33]}^{(+1)} Z_{[33]}^{(0)}\right)^{2} Z_{[33]}^{(-1)} \cdot q^{-6} A^{-12} \mathcal{F}_{10}^{(m)} & \mathcal{F}_{10}^{(n)} \\
210-1 & 210-1 \\
\hline
\end{array}
\end{aligned}
$$

for double braids and extract the $10 \times 10$ matrix $\bar{S}^{[33]}$, which is described in the appendix to this paper. Using this matrix we can calculate $H_{[3,3]}$ for some arborescent knots, which can be made without the use of the second exclusive matrix $S^{[33]}$. In examples these polynomials are consistent with available Vassiliev invariants and pass other checks from the list in [103]. Building of $S$ from $\bar{S}$ with the help of (2.1) and thus extension to arbitrary arborescent knots by the method of $[36,37,94]$ is also straightforward.

For further generalizations to other rectangular representations we need to look at the above requirements for $\mathcal{F}$ a little closer. Since it is still a guesswork, it is not really formalized - thus we provide just some sketchy comments, followed by new conjectures.

\subsection{On nullification for the unknot}

Identities, necessary for nullification of $\mathcal{F}$ for the unknot, i.e.at $m=0$ are rather simple and already the first examples reveal their general structure: if $D_{a}=\left\{A q^{a}\right\} /\{q\}=[N+a]$, then

$$
\begin{array}{r}
D_{4} D_{3}-[3] D_{4} D_{1}+[3] D_{3} D_{0}-D_{1} D_{0}=0 \\
D_{2} D_{-2}-(\underbrace{[3]+1}_{[2]^{2}}) D_{1} D_{-1}+\underbrace{\frac{[3]}{[2]} D_{0} D_{-1}+\frac{[3]}{[2]} D_{1} D_{0}}_{[3] D_{0}^{2}}=0
\end{array}
$$

3210

$$
D_{6} D_{5} D_{4}-[4] D_{6} D_{5} D_{1}+\frac{[4][3]}{[2]} D_{6} D_{3} D_{0}-[4] D_{5} D_{1} D_{0}+D_{2} D_{1} D_{0}=0
$$




$$
\begin{aligned}
& D_{4} D_{3} D_{-2}-[4] D_{4} D_{1} D_{-1}+[4] D_{3} D_{0} D_{-1}+\frac{[4]}{[2]} D_{4} D_{1} D_{0} \\
& \underbrace{-\frac{[4]}{[3]} D_{1} D_{0} D_{-1}-\frac{[4][2]}{[3]} D_{3} D_{1} D_{-1}}_{-[4] D_{2} D_{1} D_{-1}}+D_{2} D_{0} D_{-1}=0
\end{aligned}
$$

They are the first of the necessary ones for polynomiality of $\mathcal{F}$ at all $m$ - for that purpose above combinations should be proportional to the product of $D$-factors in denominators of $\mathcal{F}$, but these products have a higher degree in $A$, thus the proportionality coefficient is just zero.

Identities (7.12) are linear combinations of

$$
\begin{array}{r}
D_{a+b}+D_{a-b}=\frac{[2 b]}{[b]} D_{a}=\left(q^{b}+q^{-b}\right) D_{a} \\
{[b+c][b-c] D_{a} D_{-a}+[c+a][c-a] D_{b} D_{-b}+[a+b][a-b] D_{c} D_{-c}=0}
\end{array}
$$

which are the $q$-deformations of identities

$$
\begin{aligned}
& \sum_{i=1}^{3}\left(N+a_{i}\right) \cdot\left(a_{i+1}-a_{i+2}\right)=-\sum_{i=1}^{3}\left(N+a_{i}\right) \cdot \operatorname{det}\left(\begin{array}{cc}
1 & 1 \\
a_{i+1} & a_{i+2}
\end{array}\right)=0, \\
& \sum_{i=1}^{4}(-)^{i} \cdot\left(N+a_{i}\right)\left(N+b_{i}\right) \cdot \operatorname{det}\left(\begin{array}{ccc}
1 & 1 & 1 \\
a_{i+1}+b_{i+1} & a_{i+2}+b_{i+2} & a_{i+3}+b_{i+3} \\
a_{i+1} \cdot b_{i+1} & a_{i+2} \cdot b_{i+2} & a_{i+3} \cdot b_{i+3}
\end{array}\right)=0 \\
& \sum_{i=1}^{n+2}(-)^{(n+1) i} \underbrace{\prod_{j=1}^{n}\left(N+a_{j}^{(i)}\right)}_{N^{n}+\sum_{k=1}^{n} N^{n-k} \mu_{k}^{(i)}} \cdot \operatorname{det}_{(n+1) \times(n+1)}\left(\begin{array}{ccccc}
1 & 1 & 1 & \ldots & 1 \\
\mu_{1}^{(i+1)} & \mu_{1}^{(i+2)} & \mu_{1}^{(i+3)} & \ldots & \mu_{1}^{(i+n+1)} \\
\mu_{2}^{(i+1)} & \mu_{2}^{(i+2)} & \mu_{2}^{(i+3)} & \ldots & \mu_{2}^{(i+n+1)} \\
\ldots & & & & \\
\mu_{n}^{(i+1)} & \mu_{n}^{(i+2)} & \mu_{n}^{(i+3)} & \ldots & \mu_{n}^{(i+n+1)}
\end{array}\right)=0
\end{aligned}
$$

where $\mu^{(i+n+1)}=\mu^{(i)}$. These follow from the vanishing of $\operatorname{det}_{(n+2) \times(n+2)} P_{i}\left(x_{i^{\prime}}\right)$ for any $n+2$ polynomials of degree $n$ - because this determinant has degree $n$ in each variable $x_{i}$, while Vandermonde $\prod_{i^{\prime}<i^{\prime \prime}}^{n+2}\left(x_{i^{\prime}}-x_{i^{\prime \prime}}\right)$, to which it is obviously proportional, has bigger degree $n+1$. For our purposes we need peculiar collections of shifts $a_{j}^{(i)}$, when determinants in (7.14) are actually binomial coefficients.

Note, however, that nullification of $\mathcal{F}$ for the unknot is not by itself enough restrictive. For example, as indicated in (7.12), it would allow to change the two underlined terms in (7.8) for a single $-\frac{[4] \lambda_{03}^{2 m}}{\left\{A q^{4}\right\}\left\{A q^{3}\right\}\{A\}\left\{A / q^{2}\right\}}$ - this, however, would give a wrong (and in fact, non-polynomial) answer already for the trefoil $3_{1}$.

\subsection{Eigenvalues}

In general the eigenvalues are parameterized as

$$
\lambda_{I, J}=\prod_{f=1}^{F}\left(-q^{i_{f}-j_{f}} A\right)^{i_{f}+j_{f}+1}
$$


with $0 \leq i_{f} \leq a_{f}$ and $0 \leq j_{f} \leq b_{f}$ and an additional embedding constraint

$$
i_{f+1}<i_{f} \leq a_{f}, \quad j_{f+1}<j_{f} \leq b_{f}
$$

Also some upper floors $f$ can be left empty, i.e. allowed is also $i_{f}+j_{f}+1=0$. In particular, $\lambda_{0}=1$ is associated with all empty floors, thus it is more natural to call it $\lambda_{\emptyset}$. In this sense all eigenvalues are associated with the ordered-by-(7.16) collection of boxes in the pyramids - two per each floor $f$, except for degenerate cases when $i_{f}+j_{f}=0$ or $i_{f}+j_{f}+1=0$, and the corresponding boxes coincide or are just absent. This implies an obvious change of notation for the eigenvalue labeling.

Generic eigenvalue is labeled by a new pyramid: $\lambda_{\mathcal{I}, \mathcal{J}}=\lambda_{i_{f} j_{f}}$, and its contribution to $\mathcal{F}_{\mathcal{A}, \mathcal{B}}$ contains a product of inverse differentials $D_{k}^{-1} \sim\left\{A q^{k}\right\}^{-1}$, which only slightly depends on $\mathcal{A}$ and $\mathcal{B}$, and a combinatorial factor, accounting for the embedding of pyramids $\{\mathcal{I}, \mathcal{J}\} \subset\{\mathcal{A}, \mathcal{B}\}$.

In these pyramid notation the eigenvalues (7.5) and (7.6) fit into the big tower:

$$
\begin{aligned}
& \lambda_{\emptyset}=\lambda_{0}=1, \\
& \lambda_{0}{ }_{0}=\lambda_{1}=\lambda_{-1}=-A, \\
& \lambda_{10}=\lambda_{2}=q^{2} A^{2}, \quad \lambda_{0}{ }_{1}=\lambda_{-2}=q^{-2} A^{2}, \\
& \lambda_{20}=\lambda_{+3}=-q^{6} A^{3}, \quad \lambda_{1}{ }_{1}=\lambda_{03}=-A^{3}, \quad \lambda_{02}=\lambda_{-3}=-q^{-6} A^{3} \\
& \lambda_{30}=q^{12} A^{4}, \quad \lambda_{2}{ }_{1}=\lambda_{+_{0} 4}=q^{4} A^{4}, \quad \lambda_{00}=\lambda_{04}=A^{4}, \quad \lambda_{12}=q^{-4} A^{4}, \quad \lambda_{03}=q^{-12} A^{4} \\
& \lambda_{40}=-q^{20} A^{5}, \quad \lambda_{3}{ }_{1}=-q^{10} A^{5}, \quad \lambda_{0}{ }_{1}=\lambda_{+0} 5=-q^{4} A^{5}, \quad \lambda_{2}=-A^{5}, \quad \lambda_{0}{ }_{2}=-q^{-4} A^{5}, \quad \ldots \\
& \lambda_{5}{ }_{0}=q^{30} A^{6}, \quad \ldots \quad \lambda_{2}{ }_{1}=\lambda_{+_{0} 6}=q^{6} A^{6}, \quad \ldots
\end{aligned}
$$

\subsection{Single-floor pyramids}

Looking at a few explicit examples that we already possess, one can assume the following structure for denominators, associated with particular eigenvalues:

$$
\begin{aligned}
& \frac{\lambda_{\emptyset}^{2 m}}{\prod_{(i, j) \in(\mathcal{A}, \mathcal{B})}\left\{A q^{i-j}\right\}} \Longrightarrow \frac{\lambda_{\emptyset}^{2 m}}{\left\{A q^{a}\right\} \ldots\{A q\}\{A\}\{A / q\} \ldots\left\{A / q^{b}\right\}}
\end{aligned}
$$

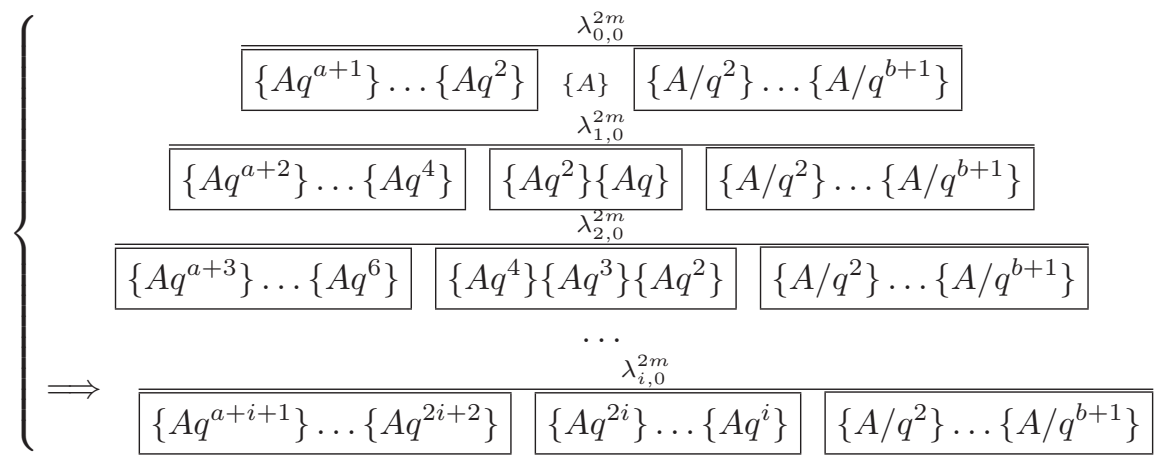




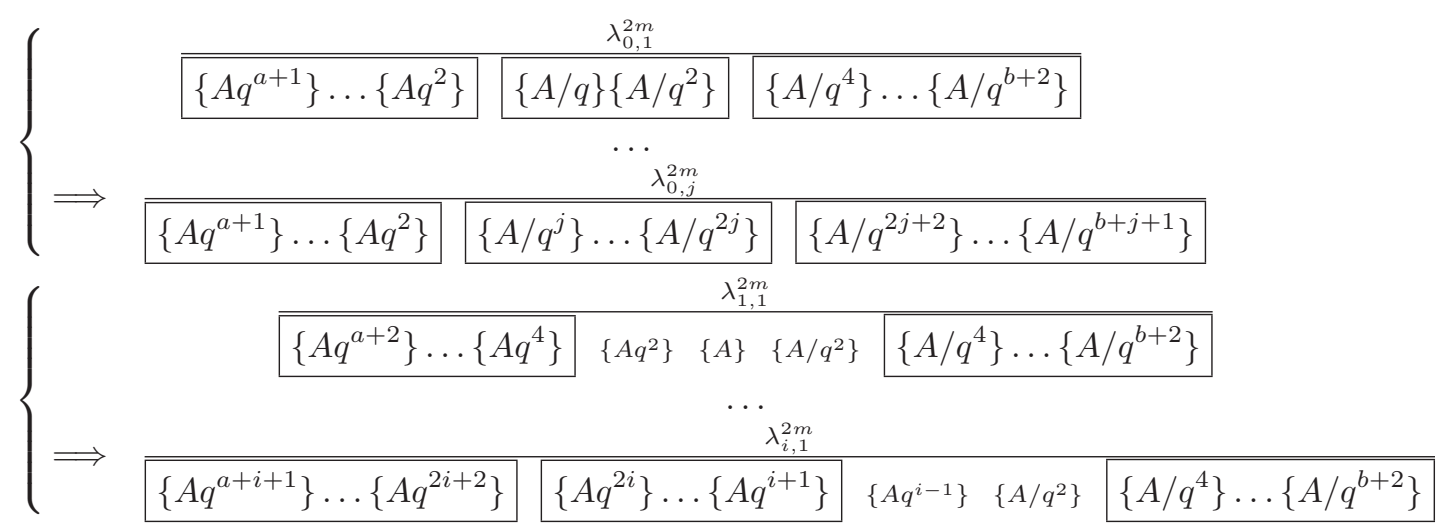

Boxes contain products $\prod_{k}\left\{A q^{k}\right\}$ over $k$ with no gaps. They turn into unity when the lower limit exceeds the upper one, like $i+1>2 i$ for $i=0$ or $2 i+2>a+i+1$ for $i=a$.

Generalization to arbitrary $\lambda_{i, j}$, appearing in the single-floor pyramids, is now obvious. More complicated is adjusting the combinatorial coefficients, which guarantee cancelation of poles and matching with the unknot, figure eight and trefoil. By trial and error we get the conjectural answer for arbitrary single-floor pyramid:

$$
\begin{aligned}
& \mathcal{F}_{\underline{a}(m)}^{(m \ldots 0 \ldots-b} \stackrel{?}{=}\left(q^{\frac{a-b}{2}} A\right)^{a+b+1}\left(\frac{1}{\sqrt{\left\{A q^{a}\right\} \ldots\left\{A / q^{b}\right\}}}+\right.
\end{aligned}
$$

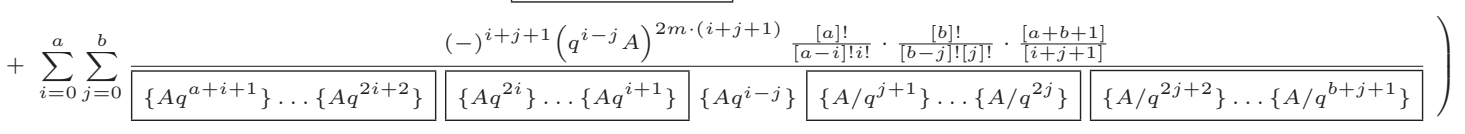

which fits all the expectations. The item in the first line contains $\lambda_{\emptyset}^{2 m}=1$ and can be considered as associated with the zeroth floor. This item is directly read from the pyramid itself, the eigenvalue leaves it intact.

\subsection{Other floors}

A natural generalization of peculiar $A$-dependent factor in front of the power of the eigenvalue $\lambda_{\substack{i_{f} \ldots j_{f} \\ i_{1} \ldots j_{1}}}$ in the multi-floor analogue of (7.18) is

$$
\frac{\left\{A q^{2 i+1}\right\}\left\{A / q^{2 j+1}\right\}}{\left\{A q^{i-j}\right\}} \longrightarrow \prod_{f^{\prime} \leq f^{\prime \prime}} \frac{\left\{A q^{i_{f^{\prime}}+i_{f^{\prime \prime}}+1}\right\}\left\{A / q^{j_{f^{\prime}}+j_{f^{\prime \prime}}+1}\right\}}{\left\{A q^{i_{f^{\prime}}-j_{f^{\prime \prime}}}\right\}\left\{A q^{i_{f^{\prime \prime}}-j_{f^{\prime}}}\right\}} \cdot \prod_{f} \frac{\left\{A q^{2 i_{f}+1}\right\}\left\{A / q^{2 j_{f}+1}\right\}}{\left\{A q^{i_{f}-j_{f}}\right\}}
$$

This gives the underlined "inter-floor interaction" factors like

$$
\begin{aligned}
\lambda_{0,0} & \longrightarrow \frac{D_{2} D_{-2}}{D_{1} D_{-1}} \\
\lambda_{0,0} & \longrightarrow \frac{D_{3} D_{-2}}{D_{2} D_{-1}} \\
2_{1} & \\
\lambda_{1,0} & \longrightarrow \frac{D_{4} D_{-2}}{D_{2} D_{0}} \\
\ldots &
\end{aligned}
$$


Note, that they all preserve parity of powers in $q$. Moreover, in empty cases, when $i_{f^{\prime \prime}}+$ $j_{f^{\prime \prime}}+1=0$ the underlined interaction factor drops away, because of the cancelations between the numerator and denominator of (7.19).

Unfortunately, this is not exactly what needed - even if a floor in the eigenvalue is empty, it still affects the shape of the contribution. This is already seen in the fully reliable (not just conjectured) formulas (7.4): the non-trivial second-floor eigenvalue $\lambda_{04}=\lambda_{0} 0$ appears only in the last term of $\mathcal{F}_{0_{0}}$, but the two other terms - with the pure-first-floor eigenvalues $\lambda_{2}=\lambda_{10}$ and $\lambda_{-2}=\frac{10-1}{=\lambda_{01}}$ are also not just the same as in $\mathcal{F} \frac{10-1}{10}$.

Another way to suspect that the empty floor matters, is to compare the first two terms in

$$
\mathcal{F}_{210-1}=q^{2} A^{4} \cdot\left(\frac{1}{\left\{A q^{2}\right\}\{A q\}\{A\}\{A / q\}}-\frac{[2]^{2} \cdot \lambda_{0,0}^{2 m}}{\left\{A q^{3}\right\}\left\{A q^{2}\right\}\{A\}\left\{A / q^{2}\right\}}+\ldots\right)
$$

and the naive

$$
\mathcal{F}_{\frac{\mathcal{F}_{0}}{210-1}} \stackrel{? ? ?}{\sim} q^{2} A^{5} \cdot\left(\frac{1}{\left\{A q^{2}\right\}\{A q\}\{A\}^{2}\{A / q\}}-\frac{c \cdot \lambda_{0,0}^{2 m}}{\left\{A q^{3}\right\}\left\{A q^{2}\right\}\{A\}^{2}\left\{A / q^{2}\right\}}+\ldots\right)
$$

The coefficient $c$ in (7.22) should be a quantization of 5 , but it should contain odd powers of $q$, thus it can not be [5]. In (7.21) its analogue is a quantization of 4 , which is made out of even powers - and it is $[2]^{2}$. However, there is no such simple way out for $c$ in $(7.22)$ - and already the second term in this formula is a less-naive combination of inverse differentials. Formula (7.9) is an example. In general, when one proceeds from $\mathcal{F}^{(m)}$ to $\mathcal{F}^{(m)} \quad$ the coefficient in front of $\lambda_{00}^{2 m}$ changes from $[a+b+1] D_{1} D_{-1}$ to $0 \ldots 0 \ldots-b$
$a \ldots 0 \ldots-b$

a sum of two items: $[a+b+1] D_{1} D_{-1}+D_{a} D_{-b}$.

This demonstrates that (7.19) is not the whole story in the multi-floor situation, that additional guesses are needed - and in fact easy to make. Still, we prefer to postpone further speculations about the higher-floor contributions, waiting for independent examination of the already made sequence of conjectures.

\section{Conclusion}

To conclude, in this paper we made a new series of conjectures, which hopefully lead to explicit formulas for exclusive Racah matrices $S$ and $\bar{S}$ and thus to construction of arborescent knot polynomials in arbitrary rectangular representations. This is a long standing problem, and its solution seems now within reach. After the structure of differential expansion for defect-zero knots (6.3) is revealed in this case in [105] and after the discovery of further factorization (6.2) of its coefficients for double braids in the present paper, it remains to conjecture these coefficients for just a relatively simple family of twist knots. To demonstrate that this can actually be possible, we provided explicit expressions for all the 
contributions (7.3)-(7.10) in the case of the previously unknown representation $R=$ [33] and made a general conjecture (7.18) for the contributions for single-floor pyramids. Its further extension to the second floor would provide the answers for arbitrary arborescent knots in representations $R=[r r]$ and $R=\left[2^{r}\right]$, while generic rectangular $R=\left[r^{s}\right]$ requires $\min (r, s)$ floors, which will hopefully come as direct generalization. Hopefully, this last step will be made soon enough.

\section{Acknowledgments}

This work was performed at the Institute for the Information Transmission Problems with the support from the Russian Science Foundation, Grant No.14-50-00150.

\section{A Exclusive Racah matrix $\bar{S}^{[3,3]}$}

The Young diagram $R=$ [33] is not symmetric and there is no additional symmetry in Racah matrix: it is just symmetric $\bar{S}_{i j}=\bar{S}_{j i}$. The transformation $\bar{S}_{i j} \longrightarrow \pm \bar{S}_{11-j, 11-i}$ converts it into something else.

Standing in the first line/column are the square roots of quantum dimensions of the ten irreducible representations, which constitute the product $[33] \otimes[\overline{[33]}$, of which only six were present in the case of $[22] \otimes \overline{[22]}$ :

$$
\begin{aligned}
d_{[33]} \bar{S}_{11} & =\sqrt{\bar{d}_{\emptyset}} & d_{\emptyset} & =1 \\
d_{[33]} \bar{S}_{21} & =\sqrt{\bar{d}_{00}} & d_{00} & =D_{1} D_{-1} \\
d_{[33]} \bar{S}_{31} & =\sqrt{\bar{d}_{10}} & \bar{d}_{10} & =\frac{D_{3} D_{0}^{2} D_{-1}}{[2]^{2}} \\
d_{[33]} \bar{S}_{41} & =\sqrt{\bar{d}_{01}} & \bar{d}_{01} & =\frac{D_{1} D_{0}^{2} D_{-3}}{[2]^{2}} \\
d_{[33]} \bar{S}_{51} & =\sqrt{\bar{d}_{11}} & \bar{d}_{11} & =\frac{D_{3} D_{1}^{2} D_{-1}^{2} D_{-3}}{[3]^{2}} \\
d_{[33]} \bar{S}_{61} & =\sqrt{\bar{d}_{20}} & \bar{d}_{20} & =\frac{D_{5} D_{1}^{2} D_{0}^{2} D_{-1}}{[3]^{2}[2]^{2}} \\
d_{[33]} \bar{S}_{71} & =\sqrt{\bar{d}_{00}} & \bar{d}_{00} & =\frac{D_{3} D_{2}^{2} D_{1} D_{-1} D_{-2}^{2} D_{-3}}{[3]^{2}[2]^{4}} \\
d_{[33]} \bar{S}_{81} & =\sqrt{\bar{d}_{21}} & \bar{d}_{21} & =\frac{D_{5} D_{2}^{2} D_{0}^{2} D_{-1}^{2} D_{-3}}{[4]^{2}[2]^{2}} \\
d_{[33]} \bar{S}_{91} & =\sqrt{\bar{d}_{00}} & \bar{d}_{20} & =\frac{D_{5} D_{3}^{2} D_{1} D_{0}^{2} D_{-1} D_{-2}^{2} D_{-3}}{[4]^{2}[3]^{2}[2]^{2}} \\
d_{[33]} \bar{S}_{10,1} & =\sqrt{\bar{d}_{10}} & \bar{d}_{21} & =\frac{D_{5} D_{4}^{2} D_{3} D_{0}^{2} D_{-1}^{3} D_{-2}^{2} D_{-3}}{[4]^{2}[3]^{4}[2]^{4}}
\end{aligned}
$$

Most other entries are not fully factorizable and non-factorized pieces can be expressed through $D_{i}=\left\{A q^{i}\right\} /\{q\}$ in different ways. The choices below are economic (with the exception of $\left.\bar{S}_{55}\right)$, but not canonical: 


$$
\begin{aligned}
& d_{[33]} \bar{S}_{12}=d_{[33]} \bar{S}_{21} \\
& d_{[33]} \bar{S}_{22}=\frac{D_{1} D_{-1}}{[3][2] D_{3} D_{-2}} \cdot\left(\left([4] D_{1}+D_{2}\right) D_{0}-[3]^{2}[2]^{2}\right) \\
& d_{[33]} \bar{S}_{32}=\frac{\sqrt{D_{1} D_{0} D_{-1}}}{[3][2] D_{2} D_{-2} \sqrt{D_{3}}} \cdot\left(\frac{[4]}{[2]} D_{1} D_{0}-[3]^{2}[2]\right) \\
& d_{[33]} \bar{S}_{42}=\frac{D_{1} D_{0} \sqrt{D_{-3} D_{-1}}}{D_{3} D_{-2}} \cdot\left(D_{2} D_{0}-[3]^{2}\right) \\
& d_{[33]} \bar{S}_{52}=\frac{D_{1} D_{-1} \sqrt{D_{1} D_{-1} D_{-3}}}{[3][2] D_{-2} \sqrt{D_{3}}} \cdot\left(D_{2} D_{0}-[3][2]^{2}\right) \\
& d_{[33]} \bar{S}_{62}=\frac{D_{1} D_{0} D_{-1} \sqrt{D_{5} D_{1}}}{[3][2]^{2} D_{3} D_{-2}} \cdot\left(D_{0}^{2}-[3][2]^{2}\right) \\
& d_{[33]} \bar{S}_{72}=\frac{D_{2} D_{1} D_{-1} \sqrt{D_{-3}}}{[3]^{2}[2]^{2} \sqrt{D_{3}}} \cdot\left(D_{3} D_{0}-[3]^{2}[2]\right) \\
& d_{[33]} \bar{S}_{82}=\frac{D_{2} D_{0} D_{-1} \sqrt{D_{5} D_{1} D_{-1} D_{-3}}}{[4][3][2] D_{3} D_{-2}} \cdot\left(D_{1} D_{0}-[3]^{2}[2]\right) \\
& d_{[33]} \bar{S}_{92}=\frac{D_{2} D_{1} D_{0} D_{-1} \sqrt{D_{5} D_{-3}}}{[4][3]^{2}[2]^{2} D_{2}} \cdot\left(D_{2} D_{0}-[3]^{2}[2]^{2}\right) \\
& d_{[33]} \bar{S}_{10,2}=-\frac{D_{4} D_{0} D_{-1}^{2} \sqrt{D_{5} D_{1} D_{-3}}}{[4][3][2] \sqrt{D_{3}}} \\
& d_{[33]} \bar{S}_{13}=d_{[33]} \bar{S}_{31} \\
& d_{[33]} \bar{S}_{23}=d_{[33]} \bar{S}_{32} \\
& d_{[33]} \bar{S}_{33}=\frac{D_{0}^{2}}{[3]^{2}[2] D_{4} D_{-2}} \cdot\left(\frac{[4]}{[2]} D_{4} D_{3} D_{-1} D_{-3}-[4][2] D_{4} D_{-1}+[3]^{2}[2]\right) \\
& d_{[33]} \bar{S}_{43}=\frac{D_{0}^{2} \sqrt{D_{1} D_{-1} D_{-3}}}{[3][2]^{2} D_{-2} \sqrt{D_{3}}} \cdot\left(D_{3} D_{0}-[4][2]^{2}\right) \\
& d_{[33]} \bar{S}_{53}=\frac{D_{1} D_{0} \sqrt{D_{-1} D_{-3}}}{[3]^{2}[2]^{2} D_{4} D_{-2}} \cdot\left(D_{5} D_{1} D_{0} D_{-1}-[3]^{2}\left([2] D_{4}+D_{3}\right) D_{0}+[4][3]^{2}[2]^{2}\right) \\
& d_{[33]} \bar{S}_{63}=\frac{D_{1} D_{0}^{2} \sqrt{D_{5}}}{[3]^{2}[2]^{2} D_{4} D_{-2} \sqrt{D_{3}}} \cdot\left(D_{4} D_{1} D_{0} D_{-3}-[2]^{3} D_{1} D_{0}+[3]^{3}[2]^{2}\right) \\
& d_{[33]} \bar{S}_{73}=-\frac{D_{2} D_{0} \sqrt{D_{1} D_{-3}}}{[3][2] D_{4}} \cdot\left(D_{4} D_{0}-\frac{[4][3]}{[2]}\right) \\
& d_{[33]} \bar{S}_{83}=\frac{D_{2} D_{0}^{2} \sqrt{D_{5} D_{-1} D_{-3}}}{[4][3]^{2}[2]^{2} D_{4} D_{-2} \sqrt{D_{3}}} \cdot\left(D_{3} D_{0}-[4][2]^{2}\right)\left(D_{1} D_{0}-[3]^{2}[2]\right) \\
& d_{[33]} \bar{S}_{93}=-\frac{D_{0}^{2} \sqrt{D_{5} D_{3} D_{1} D_{-3}}}{[4][3][2] D_{4}} \cdot\left(D_{3} D_{0}-[4][2]^{2}\right) \\
& d_{[33]} \bar{S}_{10,3}=\frac{D_{0}^{2} D_{-1} \sqrt{D_{5} D_{-3}}}{[4][2]} \\
& d_{[33]} \bar{S}_{14}=d_{[33]} \bar{S}_{41} \\
& d_{[33]} \bar{S}_{24}=d_{[33]} \bar{S}_{42} \\
& d_{[33]} \bar{S}_{34}=d_{[33]} \bar{S}_{43} \\
& d_{[33]} \bar{S}_{44}=\frac{D_{1} D_{0}^{2}}{[4][3][2] D_{3} D_{2} D_{-2}} \cdot\left([3] D_{4} D_{3} D_{-2} D_{-3}-[2] D_{2} D_{-1}+[4][3][2]\right)
\end{aligned}
$$




$$
\begin{aligned}
& d_{[33]} \bar{S}_{54}=\frac{D_{1} D_{0} D_{-1} \sqrt{D_{1}}}{[4][3][2] D_{2} D_{-2} \sqrt{D_{3}}} \cdot\left(D_{4} D_{2} D_{-1} D_{-2}-[4]^{2} D_{1} D_{0}+[4]^{2}[2]^{3}\right) \\
& d_{[33]} \bar{S}_{64}=-\frac{[4] D_{1} D_{0}^{2} \sqrt{D_{5} D_{1} D_{-1} D_{-3}}}{[3][2]^{2} D_{3} D_{-2}} \\
& d_{[33]} \bar{S}_{74}=\frac{D_{1} D_{0} \sqrt{D_{3} D_{-1}}}{[4][3]^{2}[2]^{2} D_{3}} \cdot\left(D_{4} D_{3} D_{-1} D_{-2}-[4]^{2} D_{2} D_{0}+[4]^{2}[3][2]^{2}\right) \\
& d_{[33]} \bar{S}_{84}=-\frac{D_{0}^{2} D_{-1} \sqrt{D_{5} D_{1}}}{[3][2] D_{3} D_{-2}} \cdot\left(D_{1} D_{-1}-[3]^{2}\right) \\
& d_{[33]} \bar{S}_{94}=-\frac{D_{1} D_{0}^{2} \sqrt{D_{5} D_{-1}}}{[3]^{2}[2]^{2} D_{2}} \cdot\left(D_{2} D_{-1}-[3]^{2}[2]\right) \\
& d_{[33]} \bar{S}_{10,4}=\frac{D_{4} D_{1} D_{0}^{2} D_{-1} \sqrt{D_{5} D_{-1}}}{[3][2]^{2} D_{2} \sqrt{D_{3} D_{1}}} \\
& d_{[33]} \bar{S}_{15}=d_{[33]} \bar{S}_{51} \\
& d_{[33]} \bar{S}_{25}=d_{[33]} \bar{S}_{52} \\
& d_{[33]} \bar{S}_{35}=d_{[33]} \bar{S}_{53} \\
& d_{[33]} \bar{S}_{45}=d_{[33]} \bar{S}_{54} \\
& d_{[33]} \bar{S}_{55}=\frac{D_{1}^{2} D_{-1}}{[4][3]^{2}[2]^{2} D_{4} D_{2} D_{-2}} \cdot\left(D_{5}^{2} D_{3} D_{-3}^{2} D_{-1}-3[3]^{2} D_{5} D_{2} D_{0} D_{-3}+[3]^{2}[2]^{4} D_{3} D_{-1}-\right. \\
& \left.-[3]^{2}\left(3 q^{8}+10 q^{6}+18 q^{4}+23 q^{2}+25+23 q^{-2}+18 q^{-4}+10 q^{-6}+3 q^{-8}\right)\right) \\
& d_{[33]} \bar{S}_{65}=-\frac{[4] D_{1}^{2} D_{0} \sqrt{D_{5} D_{-1} D_{-3}}}{[3]^{2}[2]^{2} D_{4} D_{-2} \sqrt{D_{3}}} \cdot\left(D_{2} D_{0}-[3][2]^{2}\right) \\
& d_{[33]} \bar{S}_{75}=-\frac{D_{1} \sqrt{D_{1} D_{-1}}}{[4][3][2] D_{4}} \cdot\left(D_{4} D_{3} D_{0} D_{-2}-[4]^{2} D_{3} D_{0}+[4]^{2}[3][2]\right) \\
& d_{[33]} \bar{S}_{85}=-\frac{D_{1} D_{0} D_{-1} \sqrt{D_{5}}}{[3]^{2}[2]^{2} D_{4} D_{-2} \sqrt{D_{3}}} \cdot\left(D_{2} D_{1} D_{0}^{2}-[3]^{2}[2]^{2} D_{1} D_{0}+[3]^{3}[2]^{3}\right) \\
& d_{[33]} \bar{S}_{95}=\frac{D_{1} D_{0} \sqrt{D_{5} D_{3} D_{1} D_{-1}}}{[3][2] D_{4} D_{2}} \cdot\left(D_{2} D_{0}-[3][2]^{2}\right) \\
& d_{[33]} \bar{S}_{10,5}=-\frac{D_{1} D_{0} D_{-1} \sqrt{D_{5} D_{-1}}}{[3] D_{2}} \\
& d_{[33]} \bar{S}_{16}=d_{[33]} \bar{S}_{61} \\
& d_{[33]} \bar{S}_{26}=d_{[33]} \bar{S}_{62} \\
& d_{[33]} \bar{S}_{36}=d_{[33]} \bar{S}_{63} \\
& d_{[33]} \bar{S}_{46}=d_{[33]} \bar{S}_{64} \\
& d_{[33]} \bar{S}_{56}=d_{[33]} \bar{S}_{65} \\
& d_{[33]} \bar{S}_{66}=\frac{D_{1}^{2} D_{0}}{[4][3]^{2}[2]^{2} D_{4} D_{3} D_{-2}} \cdot\left(D_{5} D_{4} D_{2} D_{0} D_{-2} D_{-3}-[2]^{2} D_{5} D_{4} D_{-3} D_{-2}\right. \\
& \left.+[2]^{2} D_{2} D_{0}-[4][3][2]^{3}\right) \\
& d_{[33]} \bar{S}_{76}=\frac{[4] D_{2} D_{1} D_{0} \sqrt{D_{5} D_{1} D_{-3}}}{[3][2]^{2} D_{4} \sqrt{D_{3}}} \\
& d_{[33]} \bar{S}_{86}=-\frac{D_{2} D_{1} D_{0} \sqrt{D_{-1} D_{-3}}}{[3]^{2}[2]^{2} D_{4} D_{3} D_{-2}} \cdot\left(D_{5} D_{4} D_{-1} D_{-2}-[2] D_{3} D_{0}+[4][2]^{3}\right)
\end{aligned}
$$




$$
\begin{aligned}
& d_{[33]} \bar{S}_{96}=\frac{D_{1} D_{0} \sqrt{D_{1} D_{-3}}}{[3][2]^{2} D_{4}} \cdot\left(D_{4} D_{0}-[4][2]\right) \\
& d_{[33]} \bar{S}_{10,6}=-\frac{D_{1} D_{0} D_{-1} \sqrt{D_{-3}}}{[3][2] \sqrt{D_{3}}} \\
& d_{[33]} \bar{S}_{17}=d_{[33]} \bar{S}_{71} \\
& d_{[33]} \bar{S}_{27}=d_{[33]} \bar{S}_{72} \\
& d_{[33]} \bar{S}_{37}=d_{[33]} \bar{S}_{73} \\
& d_{[33]} \bar{S}_{47}=d_{[33]} \bar{S}_{74} \\
& d_{[33]} \bar{S}_{57}=d_{[33]} \bar{S}_{75} \\
& d_{[33]} \bar{S}_{67}=d_{[33]} \bar{S}_{76} \\
& d_{[33]} \bar{S}_{77}=\frac{D_{2} D_{1}}{[4][3][2] D_{4} D_{3}} \cdot\left([3] D_{5} D_{4} D_{-1} D_{-2}-[2] D_{3} D_{0}+[4][3][2]\right) \\
& d_{[33]} \bar{S}_{87}=\frac{D_{2} D_{0} \sqrt{D_{5} D_{1} D_{-1}}}{[3][2]^{2} D_{4} \sqrt{D_{3}}} \cdot\left(D_{1} D_{0}-[3]^{2}[2]\right) \\
& d_{[33]} \bar{S}_{97}=-\frac{D_{1} D_{0} \sqrt{D_{5}}}{[3] D_{4} \sqrt{D_{3}}} \cdot\left(D_{2} D_{0}-[3]^{2}\right) \\
& d_{[33]} \bar{S}_{10,7}=\frac{D_{0} D_{-1} \sqrt{D_{5} D_{1}}}{[2] D_{3}} \\
& d_{[33]} \bar{S}_{18}=d_{[33]} \bar{S}_{81} \\
& d_{[33]} \bar{S}_{28}=d_{[33]} \bar{S}_{82} \\
& d_{[33]} \bar{S}_{38}=d_{[33]} \bar{S}_{83} \\
& d_{[33]} \bar{S}_{48}=d_{[33]} \bar{S}_{84} \\
& d_{[33]} \bar{S}_{58}=d_{[33]} \bar{S}_{85} \\
& d_{[33]} \bar{S}_{68}=d_{[33]} \bar{S}_{86} \\
& d_{[33]} \bar{S}_{78}=d_{[33]} \bar{S}_{87} \\
& d_{[33]} \bar{S}_{88}=\frac{D_{2} D_{0} D_{-1}}{[3]^{2}[2] D_{4} D_{3} D_{-2}} \cdot\left(\frac{[4]}{[2]} D_{5} D_{3} D_{-1} D_{-2}-[4][2] D_{3} D_{-2}+[3]^{2}[2]\right) \\
& d_{[33]} \bar{S}_{98}=-\frac{D_{0} \sqrt{D_{1} D_{-1}}}{[3][2] D_{4}} \cdot\left(\frac{[4]}{[2]} D_{2} D_{1}-[3]^{2}[2]\right) \\
& d_{[33]} \bar{S}_{10,8}=\frac{D_{0} D_{-1} \sqrt{D_{-1}}}{[2] \sqrt{D_{3}}} \\
& d_{[33]} \bar{S}_{19}=d_{[33]} \bar{S}_{91} \\
& d_{[33]} \bar{S}_{29}=d_{[33]} \bar{S}_{92} \\
& d_{[33]} \bar{S}_{39}=d_{[33]} \bar{S}_{93} \\
& d_{[33]} \bar{S}_{49}=d_{[33]} \bar{S}_{94} \\
& d_{[33]} \bar{S}_{59}=d_{[33]} \bar{S}_{95} \\
& d_{[33]} \bar{S}_{69}=d_{[33]} \bar{S}_{96} \\
& d_{[33]} \bar{S}_{79}=d_{[33]} \bar{S}_{97} \\
& d_{[33]} \bar{S}_{89}=d_{[33]} \bar{S}_{98} \\
& d_{[33]} \bar{S}_{99}=\frac{D_{1} D_{0}}{[3][2] D_{4} D_{2}} \cdot\left(\left([4] D_{4}+D_{3}\right) D_{-1}-[3]^{2}\right)
\end{aligned}
$$




$$
\begin{aligned}
d_{[33]} \bar{S}_{10,9} & =-\frac{D_{0} D_{-1} \sqrt{D_{1}}}{D_{2} \sqrt{D_{3}}} \\
d_{[33]} \bar{S}_{1,10} & =d_{[33]} \bar{S}_{10,1} \\
d_{[33]} \bar{S}_{2,10} & =d_{[33]} \bar{S}_{10,2} \\
d_{[33]} \bar{S}_{3,10} & =d_{[33]} \bar{S}_{10,3} \\
d_{[33]} \bar{S}_{4,10} & =d_{[33]} \bar{S}_{10,4} \\
d_{[33]} \bar{S}_{5,10} & =d_{[33]} \bar{S}_{10,5} \\
d_{[33]} \bar{S}_{6,10} & =d_{[33]} \bar{S}_{10,6} \\
d_{[33]} \bar{S}_{7,10} & =d_{[33]} \bar{S}_{10,7} \\
d_{[33]} \bar{S}_{8,10} & =d_{[33]} \bar{S}_{10,8} \\
d_{[33]} \bar{S}_{9,10} & =d_{[33]} \bar{S}_{10,9} \\
d_{[33]} \bar{S}_{10,10} & =\frac{D_{0} D_{-1}}{D_{3} D_{2}}
\end{aligned}
$$

The complementary matrix is

$$
\bar{T}_{[3,3]}=\operatorname{diag}\left(1, \quad-A, \quad q^{2} A^{2}, \quad A^{2} / q^{2}, \quad-A^{3}, \quad-q^{6} A^{3}, \quad A^{4}, \quad q^{4} A^{4}, \quad-q^{4} A^{5}, \quad q^{6} A^{6}\right)
$$

Then the eigenvalues of the product $\bar{T}^{[3,3]} \bar{S}^{[3,3]} \bar{T}^{[3,3]}$ in (2.1) are:

$$
T_{[3,3]}^{-1}=A^{6} \cdot \operatorname{diag}\left(q^{18}, \quad-q^{14}, q^{12}, q^{8}, \quad-q^{6}, q^{2}, \quad-1, q^{-2}, \quad-q^{-6}, q^{-12}\right)
$$

and the diagonalizing matrix $S^{[3,3]}$ can be obtained by the Cramer rule, i.e. its entries are minors of the matrix $\bar{T} \bar{S} \bar{T}-T^{-1}$. Cramer rule says, that any matrix $M_{i j}$ with eigenvalues $\lambda_{i}$ is diagonalized by a matrix $V_{j k}=\operatorname{Minor}_{j m}\left(M-\lambda_{k} \cdot I\right)$ with any $m$ :

$$
\sum_{j} M_{i j} V_{j k}=\sum_{j} M_{i j} \cdot \operatorname{Minor}_{j m}\left(M-\lambda_{k} \cdot I\right) \equiv \sum_{j} \lambda_{k} \delta_{i j} \operatorname{Minor}_{j m}\left(M-\lambda_{k} \cdot I\right)=\lambda_{k} V_{i k}
$$

where the boxed equality comes from

$$
\sum_{j}\left(M-\lambda_{k} \cdot I\right)_{i j} \cdot \operatorname{Minor}_{j m}\left(M-\lambda_{k} \cdot I\right)=\delta_{i m} \operatorname{det}\left(M-\lambda_{k} \cdot I\right)=0
$$

and the r.h.s. vanishes because $\lambda_{k}$ is an eigenvalue. In our case $M=\bar{T} \bar{S} \bar{T}$ and

$$
S_{i j}=\frac{\operatorname{Minor}_{i m}\left(\bar{T} \bar{S} \bar{T}-T_{j}^{-1} \cdot I\right)}{\sigma_{j}^{(m)}}
$$

The r.h.s. is actually independent of $m$ (modulo sign factors, depending on the definition of minors). The matrix of minors is normalized by division over $\sigma_{j}^{(m)}=$ $\sqrt{\sum_{i}\left(\operatorname{Minor}_{i m}\left(\bar{T} \bar{S} \bar{T}-T_{j}^{-1} \cdot I\right)\right)^{2}}$ to make $S$ orthogonal (but not symmetric). Minors are determinants and they are relatively easy to calculate (most important — to simplify and factorize, what makes this diagonalization method most practical in our situation).

With the matrix $S$ the first thing to calculate is $H_{[3,3]}^{3_{1}}=d_{[3,3]} \cdot\left(S_{[33]} T_{[33]}^{-3} S\right)_{\emptyset \emptyset}$ for the trefoil. In this way we reproduce the right answer, which was the starting point for the entire consideration in [105] - thus closing the circle of reasoning, at least in the first previously unknown case of $R=$ [33]. 
Open Access. This article is distributed under the terms of the Creative Commons Attribution License (CC-BY 4.0), which permits any use, distribution and reproduction in any medium, provided the original author(s) and source are credited.

\section{References}

[1] J.W. Alexander, Topological invariants of knots and links, Trans. Amer. Math. Soc. 30 (1928) 275.

[2] V.F.R. Jones, Index for subfactors, Invent. Math. 72 (1983) 1.

[3] V.F.R. Jones, A polynomial invariant for knots via von Neumann algebras, Bull. Amer. Math. Soc. 12 (1985) 103.

[4] V.F.R. Jones, Hecke algebra representations of braid groups and link polynomials, Annals Math. 126 (1987) 335 [INSPIRE].

[5] L. Kauffman, State models and the Jones polynomial, Topology 26 (1987) 395.

[6] P. Freyd et al., A new polynomial invariant of knots and links, Bull. Amer. Math. Soc. 12 (1985) 239.

[7] J.H. Przytycki and K.P. Traczyk, Invariants of links of Conway type, Kobe J. Math. 4 (1987) 115.

[8] J.H. Conway, Algebraic properties, in Computational problems in abstract algebra, Proc. Conf. Oxford U.K. 1967, J. Leech ed., Pergamon Press, Oxford U.K. and New York U.S.A. (1970), pg. 329.

[9] S.-S. Chern and J. Simons, Characteristic forms and geometric invariants, Annals Math. 99 (1974) 48 [INSPIRE].

[10] E. Witten, Quantum field theory and the Jones polynomial, Commun. Math. Phys. 121 (1989) 351 [INSPIRE].

[11] M. Atiyah, The geometry and physics of knots, Cambridge University Press, Cambridge U.K. (1990).

[12] R. Gopakumar and C. Vafa, On the gauge theory/geometry correspondence, Adv. Theor. Math. Phys. 3 (1999) 1415 [hep-th/9811131] [INSPIRE].

[13] H. Ooguri and C. Vafa, Knot invariants and topological strings, Nucl. Phys. B 577 (2000) 419 [hep-th/9912123] [INSPIRE].

[14] J.M.F. Labastida, M. Mariño and C. Vafa, Knots, links and branes at large-N, JHEP 11 (2000) 007 [hep-th/0010102] [INSPIRE].

[15] K. Liu and P. Peng, On a proof of the Labastida-Marino-Ooguri-Vafa conjecture, Math. Res. Lett. 17 (2010) 493 [arXiv:1012.2635] [INSPIRE].

[16] K. Liu and P. Peng, New structure of knot invariants, J. Diff. Geom. 85 (2010) 479 [arXiv: 1012.2636] [INSPIRE].

[17] S. Gukov, A.S. Schwarz and C. Vafa, Khovanov-Rozansky homology and topological strings, Lett. Math. Phys. 74 (2005) 53 [hep-th/0412243] [INSPIRE].

[18] N. Yu. Reshetikhin and V.G. Turaev, Ribbon graphs and their invariants derived from quantum groups, Commun. Math. Phys. 127 (1990) 1 [INSPIRE].

[19] E. Guadagnini, M. Martellini and M. Mintchev, Chern-Simons field theory and quantum groups, in Proc. Clausthal, (1989), pg. 307 [INSPIRE]. 
[20] E. Guadagnini, M. Martellini and M. Mintchev, Chern-Simons holonomies and the appearance of quantum groups, Phys. Lett. B 235 (1990) 275 [INSPIRE].

[21] V.G. Turaev and O.Y. Viro, State sum invariants of 3 manifolds and quantum $6 j$ symbols, Topology 31 (1992) 865 [INSPIRE].

[22] R.K. Kaul and T.R. Govindarajan, Three-dimensional Chern-Simons theory as a theory of knots and links, Nucl. Phys. B 380 (1992) 293 [hep-th/9111063] [INSPIRE].

[23] R.K. Kaul and T.R. Govindarajan, Three-dimensional Chern-Simons theory as a theory of knots and links. 2. Multicolored links, Nucl. Phys. B 393 (1993) 392 [INSPIRE].

[24] P. Rama Devi, T.R. Govindarajan and R.K. Kaul, Three-dimensional Chern-Simons theory as a theory of knots and links. 3. Compact semisimple group, Nucl. Phys. B 402 (1993) 548 [hep-th/9212110] [INSPIRE].

[25] P. Ramadevi, T.R. Govindarajan and R.K. Kaul, Knot invariants from rational conformal field theories, Nucl. Phys. B 422 (1994) 291 [hep-th/9312215] [INSPIRE].

[26] P. Ramadevi, T.R. Govindarajan and R.K. Kaul, Representations of composite braids and invariants for mutant knots and links in Chern-Simons field theories, Mod. Phys. Lett. A 10 (1995) 1635 [hep-th/9412084] [INSPIRE].

[27] P. Ramadevi, T.R. Govindarajan and R.K. Kaul, Chirality of knots $9_{42}$ and $10_{71}$ and Chern-Simons theory, Mod. Phys. Lett. A 9 (1994) 3205 [hep-th/9401095] [INSPIRE].

[28] P. Ramadevi and T. Sarkar, On link invariants and topological string amplitudes, Nucl. Phys. B 600 (2001) 487 [hep-th/0009188] [inSPIRE].

[29] Zodinmawia and P. Ramadevi, SU(N) quantum Racah coefficients \& non-torus links, Nucl. Phys. B 870 (2013) 205 [arXiv:1107.3918] [InSPIRE].

[30] Zodinmawia and P. Ramadevi, Reformulated invariants for non-torus knots and links, arXiv: 1209.1346 [INSPIRE].

[31] A. Morozov and A. Smirnov, Chern-Simons theory in the temporal gauge and knot invariants through the universal quantum R-matrix, Nucl. Phys. B $\mathbf{8 3 5}$ (2010) 284 [arXiv: 1001.2003] [INSPIRE].

[32] A. Smirnov, Notes on Chern-Simons theory in the temporal gauge, in Proc. of International School of Subnuclear Phys., Erice Italy (2009) [Subnucl. Ser. 47 (2011) 489] [arXiv:0910.5011] [INSPIRE].

[33] D. Bar-Natan and S. Morrison, The Knot atlas webpage, http://katlas.org.

[34] C. Livingston and J.C. Cha, Table of knot invariants webpage, http://indiana.edu/ knotinfo/.

[35] Knotebook webpage, http://knotebook.org.

[36] A. Mironov and A. Morozov, Towards effective topological field theory for knots, Nucl. Phys. B 899 (2015) 395 [arXiv:1506.00339] [inSPIRE].

[37] A. Mironov, A. Morozov, A. Morozov, A. Sleptsov, P. Ramadevi and V.K. Singh, Tabulating knot polynomials for arborescent knots, arXiv:1601.04199 [INSPIRE].

[38] A.A. Belavin, A.M. Polyakov and A.B. Zamolodchikov, Infinite conformal symmetry in two-dimensional quantum field theory, Nucl. Phys. B 241 (1984) 333 [InSPIRE].

[39] A. Zamolodchikov and Al. Zamolodchikov, Conformal field theory and critical phenomena in $2 d$ systems, (2009). 
[40] V.S. Dotsenko and V.A. Fateev, Conformal algebra and multipoint correlation functions in two-dimensional statistical models, Nucl. Phys. B 240 (1984) 312 [INSPIRE].

[41] A. Gerasimov, A. Morozov, M. Olshanetsky, A. Marshakov and S.L. Shatashvili, Wess-Zumino-Witten model as a theory of free fields, Int. J. Mod. Phys. A 5 (1990) 2495 [INSPIRE].

[42] L. Álvarez-Gaumé, Random surfaces, statistical mechanics and string theory, Helv. Phys. Acta 64 (1991) 359 [INSPIRE].

[43] P. Di Francesco, P. Mathieu and D. Senechal, Conformal field theory, Springer, Germany (1996).

[44] A. Mironov, S. Mironov, A. Morozov and A. Morozov, CFT exercises for the needs of AGT, Theor. Math. Phys. 165 (2010) 1662 [Teor. Mat. Fiz. 165 (2010) 503] [arXiv:0908.2064] [INSPIRE].

[45] N.A. Nekrasov, Seiberg-Witten prepotential from instanton counting, Adv. Theor. Math. Phys. 7 (2003) 831 [hep-th/0206161] [INSPIRE].

[46] R. Flume and R. Poghossian, An algorithm for the microscopic evaluation of the coefficients of the Seiberg-Witten prepotential, Int. J. Mod. Phys. A 18 (2003) 2541 [hep-th/0208176] [INSPIRE].

[47] N. Nekrasov and A. Okounkov, Seiberg-Witten theory and random partitions, Prog. Math. 244 (2006) 525 [hep-th/0306238] [INSPIRE].

[48] N. Nekrasov and V. Pestun, Seiberg-Witten geometry of four dimensional $N=2$ quiver gauge theories, arXiv:1211.2240 [INSPIRE].

[49] N. Nekrasov, V. Pestun and S. Shatashvili, Quantum geometry and quiver gauge theories, arXiv: 1312.6689 [INSPIRE].

[50] L.F. Alday, D. Gaiotto and Y. Tachikawa, Liouville correlation functions from four-dimensional gauge theories, Lett. Math. Phys. 91 (2010) 167 [arXiv:0906.3219] [INSPIRE].

[51] N. Wyllard, $A_{N-1}$ conformal Toda field theory correlation functions from conformal $N=2$ $\mathrm{SU}(N)$ quiver gauge theories, JHEP 11 (2009) 002 [arXiv:0907.2189] [INSPIRE].

[52] A. Mironov and A. Morozov, On AGT relation in the case of $\mathrm{U}(3)$, Nucl. Phys. B 825 (2010) 1 [arXiv:0908.2569] [InSPIRE].

[53] B. Feigin, E. Feigin, M. Jimbo, T. Miwa and E. Mukhin, Quantum continuous $\mathfrak{g l}_{\infty}$ : semiinfinite construction of representations, Kyoto J. Math. 51 (2011) 337 [arXiv: 1002 .3100].

[54] B. Feigin, E. Feigin, M. Jimbo, T. Miwa and E. Mukhin, Quantum continuous $\mathfrak{g l}_{\infty}$ : tensor products of Fock modules and $\mathcal{W}_{n}$-characters, Kyoto J. Math. 51 (2011) 365 [arXiv: 1002.3113] [INSPIRE].

[55] A. Mironov, A. Morozov, S. Shakirov and A. Smirnov, Proving AGT conjecture as HS duality: extension to five dimensions, Nucl. Phys. B 855 (2012) 128 [arXiv:1105.0948] [INSPIRE].

[56] J.-E. Bourgine, Y. Matsuo and H. Zhang, Holomorphic field realization of $S H^{c}$ and quantum geometry of quiver gauge theories, JHEP 04 (2016) 167 [arXiv:1512.02492] [INSPIRE].

[57] H. Awata et al., Explicit examples of DIM constraints for network matrix models, JHEP 07 (2016) 103 [arXiv:1604.08366] [INSPIRE]. 
[58] A. Mironov, A. Morozov and An. Morozov, Character expansion for HOMFLY polynomials. I. Integrability and difference equations, in Strings, Gauge Fields, and the Geometry Behind: The Legacy of Maximilian Kreuzer, A. Rebhan, L. Katzarkov, J. Knapp, R. Rashkov and E. Scheidegger eds., World Scientific Publishing Co. Pte. Ltd., Singapore (2013), pg. 101 [arXiv:1112.5754].

[59] A. Mironov, A. Morozov and A. Morozov, Character expansion for HOMFLY polynomials. II. Fundamental representation. Up to five strands in braid, JHEP 03 (2012) 034 [arXiv: 1112.2654] [INSPIRE].

[60] A. Mironov, A. Morozov and A. Morozov, On colored HOMFLY polynomials for twist knots, Mod. Phys. Lett. A 29 (2014) 1450183 [arXiv: 1408.3076] [InSPIRE].

[61] A. Anokhina, A. Mironov, A. Morozov and A. Morozov, Racah coefficients and extended HOMFLY polynomials for all 5-, 6- and 7-strand braids, Nucl. Phys. B 868 (2013) 271 [arXiv: 1207.0279] [INSPIRE].

[62] A. Anokhina, A. Mironov, A. Morozov and A. Morozov, Knot polynomials in the first non-symmetric representation, Nucl. Phys. B 882 (2014) 171 [arXiv:1211.6375] [INSPIRE].

[63] A. Anokhina, A. Mironov, A. Morozov and A. Morozov, Colored HOMFLY polynomials as multiple sums over paths or standard Young tableaux, Adv. High Energy Phys. 2013 (2013) 931830 [arXiv:1304.1486] [INSPIRE].

[64] A. Anokhina, On R-matrix approaches to knot invariants, arXiv:1412.8444 [INSPIRE].

[65] S. Gukov and M. Stošić, Homological algebra of knots and BPS states, Proc. Symp. Pure Math. 85 (2012) 125 [arXiv:1112.0030] [INSPIRE].

[66] S. Nawata, P. Ramadevi, Zodinmawia and X. Sun, Super-A-polynomials for twist knots, JHEP 11 (2012) 157 [arXiv:1209.1409] [InSPIRE].

[67] S. Nawata, P. Ramadevi and Zodinmawia, Multiplicity-free quantum 6j-symbols for $U_{q}\left(\mathfrak{s l}_{N}\right)$, Lett. Math. Phys. 103 (2013) 1389 [arXiv:1302.5143] [INSPIRE].

[68] S. Nawata, P. Ramadevi and Zodinmawia, Colored HOMFLY polynomials from Chern-Simons theory, J. Knot Theor. 22 (2013) 1350078 [arXiv:1302.5144] [INSPIRE].

[69] S. Nawata, P. Ramadevi and Zodinmawia, Colored Kauffman homology and super-A-polynomials, JHEP 01 (2014) 126 [arXiv:1310.2240] [INSPIRE].

[70] Zodinmawia, Knot polynomials from $\mathrm{SU}(N)$ Chern-Simons theory, superpolynomials and super-A-polynomials, Ph.D. thesis, IIT, Mumbai India (2014).

[71] A. Anokhina and A. Morozov, Cabling procedure for the colored HOMFLY polynomials, Theor. Math. Phys. 178 (2014) 1 [Teor. Mat. Fiz. 178 (2014) 3] [arXiv:1307.2216] [INSPIRE].

[72] S. Nawata, P. Ramadevi and Zodinmawia, Colored Kauffman homology and super-A-polynomials, JHEP 01 (2014) 126 [arXiv:1310.2240] [INSPIRE].

[73] S. Gukov, S. Nawata, I. Saberi, M. Stošić and P. Sulkowski, Sequencing BPS spectra, JHEP 03 (2016) 004 [arXiv:1512.07883] [INSPIRE].

[74] D. Galakhov, D. Melnikov, A. Mironov, A. Morozov and A. Sleptsov, Colored knot polynomials for arbitrary pretzel knots and links, Phys. Lett. B 743 (2015) 71 [arXiv:1412.2616] [INSPIRE]. 
[75] D. Galakhov, D. Melnikov, A. Mironov and A. Morozov, Knot invariants from Virasoro related representation and pretzel knots, Nucl. Phys. B 899 (2015) 194 [arXiv:1502.02621] [INSPIRE].

[76] S. Nawata, P. Ramadevi and V.K. Singh, Colored HOMFLY polynomials can distinguish mutant knots, arXiv:1504.00364 [INSPIRE].

[77] S. Garoufalidis, A.D. Lauda and T.T.Q. Lê, The colored HOMFLYPT function is q-holonomic, arXiv:1604.08502 [INSPIRE].

[78] M. Rosso and V.F.R. Jones, On the invariants of torus knots derived from quantum groups, J. Knot Theor. 2 (1993) 97.

[79] X.-S. Lin and H. Zheng, On the Hecke algebras and the colored HOMFLY polynomial, Trans. Amer. Math. Soc. 362 (2010) 1 [math/0601267].

[80] S. Stevan, Chern-Simons invariants of torus links, Annales Henri Poincaré 11 (2010) 1201 [arXiv: 1003.2861] [INSPIRE].

[81] M. Tierz, Soft matrix models and Chern-Simons partition functions, Mod. Phys. Lett. A 19 (2004) 1365 [hep-th/0212128] [InSPIRE].

[82] A. Brini, B. Eynard and M. Mariño, Torus knots and mirror symmetry, Annales Henri Poincaré 13 (2012) 1873 [arXiv:1105.2012] [INSPIRE].

[83] A. Aleksandrov, A.D. Mironov, A. Morozov and A.A. Morozov, Towards matrix model representation of HOMFLY polynomials, JETP Lett. 100 (2014) 271 [Pisma Zh. Eksp. Teor. Fiz. 100 (2014) 297] [arXiv:1407.3754] [INSPIRE].

[84] M. Aganagic and S. Shakirov, Knot homology and refined Chern-Simons index, Commun. Math. Phys. 333 (2015) 187 [arXiv:1105.5117] [INSPIRE].

[85] M. Aganagic and S. Shakirov, Refined Chern-Simons theory and knot homology, Proc. Symp. Pure Math. 85 (2012) 3 [arXiv:1202.2489] [INSPIRE].

[86] M. Aganagic and S. Shakirov, Refined Chern-Simons theory and topological string, arXiv: 1210.2733 [INSPIRE].

[87] A. Mironov, A. Morozov, S. Shakirov and A. Sleptsov, Interplay between MacDonald and Hall-Littlewood expansions of extended torus superpolynomials, JHEP 05 (2012) 070 [arXiv: 1201.3339] [INSPIRE].

[88] P. Dunin-Barkowski, A. Mironov, A. Morozov, A. Sleptsov and A. Smirnov, Superpolynomials for toric knots from evolution induced by cut-and-join operators, JHEP 03 (2013) 021 [arXiv:1106.4305] [INSPIRE].

[89] I. Cherednik, Jones polynomials of torus knots via DAHA, arXiv:1111.6195 [INSPIRE].

[90] E. Gorsky and A. Negut, Refined knot invariants and Hilbert schemes, J. Math. Pure. Appl. 104 (2015) 403 [arXiv:1304.3328] [INSPIRE].

[91] I. Cherednik and I. Danilenko, DAHA and iterated torus knots, Algebr. Geom. Topol. 16 (2016) 843 [arXiv:1408.4348].

[92] S. Arthamonov and S. Shakirov, Refined Chern-Simons theory in genus two, arXiv: 1504.02620 [INSPIRE].

[93] E. Gorsky, S. Gukov and M. Stošić, Quadruply-graded colored homology of knots, arXiv: 1304.3481 [INSPIRE].

[94] A. Mironov, A. Morozov, A. Morozov, P. Ramadevi and V.K. Singh, Colored HOMFLY polynomials of knots presented as double fat diagrams, JHEP 07 (2015) 109 [arXiv: 1504.00371] [INSPIRE]. 
[95] A. Caudron, Classification des noeuds et des enlacements (in French), Publ. Math. Orsay 82-4, University of Paris XI, Orsay France (1982).

[96] F. Bonahon and L.C. Siebenmann, New geometric splittings of classical knots and the classification and symmetries of arborescent knots, http://www-bcf.usc.edu/ fbonahon/Research/Preprints/BonSieb.pdf, (2010).

[97] H. Itoyama, A. Mironov, A. Morozov and A. Morozov, Character expansion for HOMFLY polynomials. III. All 3-strand braids in the first symmetric representation, Int. J. Mod. Phys. A 27 (2012) 1250099 [arXiv:1204.4785] [InSPIRE].

[98] S. Nawata, P. Ramadevi, Zodinmawia and X. Sun, Super-A-polynomials for twist knots, JHEP 11 (2012) 157 [arXiv:1209.1409] [INSPIRE].

[99] H. Fuji, S. Gukov, M. Stošić and P. Sulkowski, $3 d$ analogs of Argyres-Douglas theories and knot homologies, JHEP 01 (2013) 175 [arXiv:1209.1416] [INSPIRE].

[100] A. Mironov, A. Morozov and A. Sleptsov, Colored HOMFLY polynomials for the pretzel knots and links, JHEP 07 (2015) 069 [arXiv: 1412.8432] [INSPIRE].

[101] J. Gu and H. Jockers, A note on colored HOMFLY polynomials for hyperbolic knots from WZW models, Commun. Math. Phys. 338 (2015) 393 [arXiv: 1407.5643] [inSPIRE].

[102] A. Mironov, A. Morozov, A. Morozov and A. Sleptsov, Racah matrices and hidden integrability in evolution of knots, Phys. Lett. B 760 (2016) 45 [arXiv:1605.04881] [INSPIRE].

[103] A. Mironov, A. Morozov, A. Morozov and A. Sleptsov, HOMFLY polynomials in representation [3,1] for 3-strand braids, arXiv: 1605.02313 [INSPIRE].

[104] A. Mironov, A. Morozov, A. Morozov and A. Sleptsov, Quantum Racah matrices and 3-strand braids in irreps $R$ with $|R|=4$, JETP Lett. 104 (2016) 56 [Pisma Zh. Eksp. Teor. Fiz. 104 (2016) 52] [arXiv:1605.03098] [INSPIRE].

[105] A. Morozov, Differential expansion and rectangular HOMFLY for the figure eight knot, Nucl. Phys. B 911 (2016) 582 [arXiv: 1605.09728] [inSPIRE].

[106] H. Itoyama, A. Mironov, A. Morozov and A. Morozov, HOMFLY and superpolynomials for figure eight knot in all symmetric and antisymmetric representations, JHEP 07 (2012) 131 [arXiv:1203.5978] [INSPIRE].

[107] A. Mironov, A. Morozov and A. Morozov, Evolution method and "differential hierarchy" of colored knot polynomials, AIP Conf. Proc. 1562 (2013) 123 [arXiv:1306.3197] [InSPIRE].

[108] S.B. Arthamonov, A. Mironov and A. Morozov, Differential hierarchy and additional grading of knot polynomials, Theor. Math. Phys. 179 (2014) 509 [Teor. Mat. Fiz. 179 (2014) 147] [arXiv: 1306.5682] [INSPIRE].

[109] S. Arthamonov, A. Mironov, A. Morozov and A. Morozov, Link polynomial calculus and the AENV conjecture, JHEP 04 (2014) 156 [arXiv: 1309.7984] [INSPIRE].

[110] Ya. Kononov and A. Morozov, On the defect and stability of differential expansion, JETP Lett. 101 (2015) 831 [Pisma Zh. Eksp. Teor. Fiz. 101 (2015) 931] [arXiv: 1504.07146] [INSPIRE].

[111] N.M. Dunfield, S. Gukov and J. Rasmussen, The superpolynomial for knot homologies, Exper. Math. 15 (2006) 129 [math/0505662] [INSPIRE].

[112] M. Khovanov, A categorification of the Jones polynomial, Duke Math. J. 101 (2000) 359 [math/9908171] [INSPIRE]. 
[113] M. Khovanov, Patterns in knot cohomology I, Exper. Math. 12 (2003) 365 [math/0201306].

[114] M. Khovanov, Categorifications of the colored Jones polynomial, J. Knot Theor. 14 (2005) 111 [math/0302060].

[115] M. Khovanov, sl(3) link homology, Algebr. Geom. Topol. 4 (2004) 1045 [math/0304375].

[116] M. Khovanov, Triply-graded link homology and Hochschild homology of Soergel bimodules, Int. J. Math. 18 (2007) 869 [math/0510265].

[117] M. Khovanov, Link homology and categorification, math/0605339.

[118] M. Khovanov, Categorifications from planar diagrammatics, arXiv:1008.5084.

[119] D. Bar-Natan, On Khovanov's categorification of the Jones polynomial, Algebr. Geom. Topol. 2 (2002) 337 [math/0201043].

[120] D. Bar-Natan, Khovanov's homology for tangles and cobordisms, Geom. Topol. 9 (2005) 1443 [math/0410495].

[121] D. Bar-Natan, Fast Khovanov homology computations, J. Knot Theor. 16 (2007) 243 [math/0606318].

[122] M. Khovanov and L. Rozansky, Matrix factorizations and link homology, Fund. Math. 199 (2008) 191 [math/0401268].

[123] M. Khovanov and L. Rozansky, Matrix factorizations and link homology II, Geom. Topol. 12 (2008) 1387 [math/0505056].

[124] M. Khovanov and L. Rozansky, Virtual crossings, convolutions and a categorification of the $\mathrm{SO}(2 N)$ Kauffman polynomial, math/0701333.

[125] N. Carqueville and D. Murfet, Computing Khovanov-Rozansky homology and defect fusion, Algebr. Geom. Topol. 14 (2014) 489 [arXiv:1108.1081] [INSPIRE].

[126] V. Dolotin and A. Morozov, Introduction to Khovanov homologies. I. Unreduced Jones superpolynomial, JHEP 01 (2013) 065 [arXiv: 1208.4994] [INSPIRE].

[127] V. Dolotin and A. Morozov, Introduction to Khovanov homologies. II. Reduced Jones superpolynomials, J. Phys. Conf. Ser. 411 (2013) 012013 [arXiv:1209.5109] [INSPIRE].

[128] V. Dolotin and A. Morozov, Introduction to Khovanov homologies. III. A new and simple tensor-algebra construction of Khovanov-Rozansky invariants, Nucl. Phys. B 878 (2014) 12 [arXiv: 1308.5759] [INSPIRE].

[129] E. Witten, Two lectures on the Jones polynomial and Khovanov homology, arXiv: 1401.6996 [INSPIRE].

[130] A. Anokhina and A. Morozov, Towards R-matrix construction of Khovanov-Rozansky polynomials. I. Primary T-deformation of HOMFLY, JHEP 07 (2014) 063 [arXiv: 1403.8087] [INSPIRE].

[131] S. Nawata and A. Oblomkov, Lectures on knot homology, arXiv:1510.01795 [INSPIRE]. 